\title{
Retinal pigment epithelium degeneration caused by aggregation of PRPF 31 and the role of HSP70 family of proteins
}

\author{
Lourdes Valdés-Sánchez ${ }^{1 \dagger}$, Sofia M. Calado ${ }^{1,2+}$, Berta de la Cerda ${ }^{1}$, Ana Aramburu ${ }^{1,3}$, Ana Belén García-Delgado ${ }^{1}$ \\ Simone Massalini 1,4, Adoración Montero-Sánchez' ${ }^{1}$ Vaibhav Bhatia', Eduardo Rodríguez-Bocanegra ${ }^{1,5}$, \\ Andrea Diez-Lloret ${ }^{1}$, Daniel Rodríguez-Martínez ${ }^{1}$, Christina Chakarova ${ }^{6}$, Shom S. Bhattacharya ${ }^{{ }^{*}}$ and \\ Francisco J. Díaz-Corrales ${ }^{1 *}$ (D)
}

\begin{abstract}
Background: Mutations in pre-mRNA splicing factor PRPF31 can lead to retinitis pigmentosa (RP). Although the exact disease mechanism remains unknown, it has been hypothesized that haploinsufficiency might be involved in the pathophysiology of the disease.

Methods: In this study, we have analyzed a mouse model containing the p.A216P mutation in Prpf31 gene.

Results: We found that mutant Prpf31 protein produces cytoplasmic aggregates in the retinal pigment epithelium and decreasing the protein levels of this splicing factor in the nucleus. Additionally, normal protein was recruited in insoluble aggregates when the mutant protein was overexpressed in vitro. In response to protein aggregation, Hspa4l is overexpressed. This member of the HSP70 family of chaperones might contribute to the correct folding and solubilization of the mutant protein, allowing its translocation to the nucleus.

Conclusions: Our data suggests that a mechanism haploinsufficiency and dominant-negative is involved in retinal degeneration due to mutations in PRPF31. HSP70 over-expression might be a new therapeutic target for the treatment of retinal degeneration due to PRPF31 mutations.
\end{abstract}

Keywords: HSP70, PRPF31, Retinal degeneration, Retinal pigment epithelium, Retinitis pigmentosa

\section{Background}

Retinitis pigmentosa (RP) is one of a diverse group of retinal dystrophies and one of the commonest causes of inherited blindness in adults, affecting around 1:4000 individuals worldwide (Verbakel et al., 2018). RP initially presents a progressive impairment and cell death of rod photoreceptors, followed by loss of cones and retinal pigment epithelium (RPE). Clinically, RP is characterized by

\footnotetext{
*Correspondence: shomi.bhattacharya@ucl.ac.uk; francisco.diaz@cabimer.es †Lourdes Valdés-Sánchez and Sofia M. Calado contributed equally to this work.

${ }^{6}$ Institute of Ophthalmology, University College London, 11-43 Bath Street, London EC1V 9EL, UK

${ }^{1}$ Regeneration and Cell Therapy Department, Andalusian Molecular Biology and Regenerative Medicine Centre-CABIMER (Junta de Andalucía), CSIC, Universidad de Sevilla, Universidad Pablo de Olavide, Avda. Americo Vespucio 24, 41092 Seville, Spain

Full list of author information is available at the end of the article
}

night blindness, which usually starts during adolescence and progresses with constriction of the visual field and a marked reduction in the amplitude of electroretinogram (ERG) waves. So far, mutations in more than 80 genes have been implicated in nonsyndromic RP (Verbakel et al., 2018). Many of these genes encode for retinal-specific proteins; however, some are ubiquitously expressed, such as splicing factors PRPF3, PRPF4, PRPF6, PRPF8 and PRPF31 (Liu E Zack, 2013; Ruzickova E Stanek, 2017).

Pre-mRNA splicing is a general cellular function crucial for the expression of eukaryotic transcripts. It is catalyzed by the spliceosome, a large ribonucleoprotein complex composed of five small nuclear ribonucleoprotein complexes (Ruzickova \& Stanek, 2017). In humans, PRPF31 encodes the homolog of $S$. cerevisiae premRNA processing factor 31, also known as PRPF31 
protein (Vithana et al., 2001). PRPF31 is required for the U4/U6-U5 tri-snRNP formation and spliceosome activity (Makarova et al., 2002; Schaffert et al., 2004). Mutations in PRPF31 have been described as the second most common cause of autosomal dominant RP (adRP) known as RP11 (Vithana et al., 2001; AlMaghtheh et al., 1998; Rose et al., 2016) and, although PRPF31 is necessary for pre-mRNA splicing in every cell, adRP is the only clinical entity associated with these mutations.

Curiously, within the PRPF31-affected families, it is common to find asymptomatic carriers due to overexpression of the WT allele inherited from the normal parent. Therefore, the differential expression of the WT allele explains the incomplete penetrance associated with this RP locus (Rose et al., 2016; Vithana et al., 2003). It has recently been described that the expression level of PRPF31 is regulated by the number of copies of a minisatellite repeat element-MSR1 located $200 \mathrm{bp}$ upstream of the promoter. High-expressing WT alleles are found in asymptomatic carriers and low-expressing alleles are associated with the disease, where the amount of WT PRPF31 protein produced is beneath its threshold for normal function (Rose et al., 2016).

Although haploinsufficiency contributes to the physiopathology of the disease, it is still not clear how retinal degeneration occurs in patients carrying PRPF31 mutations. To explore disease mechanisms, two animal models were previously generated (Bujakowska et al., 2009). One was a heterozygous knockout (KO) mouse $\left(\operatorname{Prpf} 31^{+/-}\right)$and the second a knock-in (KI) mouse carrying the point mutation p.A216P $\left(\operatorname{Prpf3} 1^{A 216 P /+}\right)$. This mutation was previously identified in RP11 patients with a severe retinal phenotype (Vithana et al., 2001). However, both heterozygous mouse models did not show any sign of photoreceptor degeneration and, as expected, the homozygous mutant mice were found to be embryonic lethal (Bujakowska et al., 2009). Based on these results, it was speculated that Prpf31 is essential for survival and the presence of one WT Prpf31 allele is enough to maintain retinal function with no dominant-negative effect of the p.A216P mutation in mice.

More recently, it has been published that three splicing-factor mouse models (Prpf3 ${ }^{\text {T494M/+ }}$, $\operatorname{Prpf}^{\mathrm{H} 2309 \mathrm{P} /+}$ and $\mathrm{Prpf}_{31^{+/-}}$) develop late-onset morphological changes and dysfunction in the RPE rather than photoreceptor degeneration (Farkas et al., 2014; Graziotto et al., 2011). Therefore, in this work, we decided to study the effect of the p.A216P mutation on RPE. We found mislocalization and aggregation of the mutant Prpf31 protein with concomitant depletion of normal protein. These results indicate mixed haploinsufficiency and dominantnegative mechanisms involved in retinal degeneration due to mutations in PRPF31. Also, this work postulates HSP70 modulation as a new therapeutic target for the treatment of RP due to PRPF31 mutations.

\section{Methods \\ Animal handling and eye samples}

Eight to sixteen-month old C57BL/6 J Prpf31 ${ }^{+/+}$(WT) and C57BL/6 J Prpf31 $1^{A 216 P /+}$ (KI) mice were housed in the Biological Resources Unit of CABIMER and kept in a temperature-controlled environment $\left(21 \pm 1^{\circ} \mathrm{C}\right)$, with a relative humidity of $55 \pm 5 \%$, a light/dark cycle $08: 00-20$ : 00 and given standard mouse chow and water ad libitum. Mouse genotyping was performed as previously described (Bujakowska et al., 2009). Due to homozygous Prpf31 ${ }^{A 216 P / A 216 P}$ mice are not viable, we use $\operatorname{Prpf}_{31^{A 216 P /+}}$ and $\operatorname{Prpf} \mathrm{1}^{+/+}$mice to obtain a similar proportion of KI and WT in each litter. The WT mice used as controls in each experiment belonged to the same litter of the $\operatorname{Prpf} 31^{A 216 P /+}$ mice. The $r d 8$ mutants were discarded in these mice using the specific primers: forward 5'-GCC CCT GTT TGC ATG GAG GAA ACT TGG AAG ACA GTC ACA GTT CTT CTG-3' and reverse 5'-GCC CCA TTT GCA CAC TGA TGA C-3' (Mattapallil et al., 2012). A group of WT CD-1 mice were also used for the immunohistochemistry experiments.

All experiments described in this work were performed in compliance with the Spanish and European Laboratory Animal Science Association-FELASA Guide for the Care and Use of Laboratory Animals, the European Union Council Directive 2010/63/EU for the use of animals and the Association for Research in Vision and Ophthalmology-ARVO for the use of animals in ophthalmic and vision research. Animal manipulation and experimental methods have been approved by the Ethics Committee for Animal Experimentation of CABIMER, Seville, Spain. All efforts were made to minimize the number of animals used and their suffering. Pig and cow eye samples were obtained from a local slaughterhouse. Human eye sample for Western blotting was obtained from a deceased healthy donor, in a procedure approved by the Ethics Committee of University Hospital Virgen Macarena, Seville, Spain.

\section{Immunohistochemistry and immunofluorescence experiments}

Immunohistochemistry was performed to evaluate distribution of Prpf 31 protein in retinal sections of WT CD-1 mice. The animals were euthanized by cervical dislocation and the eyes excised quickly and fixed in ice-cold $4 \%$ paraformaldehyde (PFA) in PBS, overnight, at $4{ }^{\circ} \mathrm{C}$. The fixed eyes were then cryoprotected in $30 \%$ sucrose in PBS, and embedded in optimal cutting temperature compound for cryotome sections. Serial sections of $18 \mu \mathrm{m}$ thick were mounted in five parallel series and processed for immunohistochemistry. Briefly, the retinal 
sections were kept in $3 \% \mathrm{H}_{2} \mathrm{O}_{2}$ in PBS for $30 \mathrm{~min}$. Samples were then washed in $0.2 \%$ Triton X-100/PBS (PBS$\mathrm{T})$ and blocked in 1\% BSA/PBS-T at room temperature for $1 \mathrm{~h}$. Incubation with the primary antibody goat antiPRPF31 (1:100; OriGene Technologies Inc., Maryland, USA, TA302582) and mouse anti-Rhodopsin (1:1000; Abcam, Cambridge, UK, ab190307) were performed overnight at $4{ }^{\circ} \mathrm{C}$. After incubation, samples were washed 3 times in PBS-T, and incubated with appropriate biotinylated anti-goat IgG (1:500; Vector Laboratories, California, USA, BA9500) and anti-mouse IgG (1:250; Chemicon International, California, USA, AP124B) antibodies for $1 \mathrm{~h}$ at room temperature. The retinal sections were incubated for $1 \mathrm{~h}$ in avidin-biotin-peroxidase complex (1:500; Vector Laboratories). The immuno-reactive signals were visualized by $0.02 \% 3,3^{\prime}$-diaminobenzidine, $0.4 \%$ nickel ammonium sulphate and $0.005 \% \mathrm{H}_{2} \mathrm{O}_{2}$ in $50 \mathrm{nM}$ Tris- $\mathrm{HCl}$ buffer. Standard haematoxylin staining was performed in order to observe cell nuclei in the retinal samples. Finally, samples were dehydrated and mounted with Eukitt mounting medium (Sigma-Aldrich, Missouri, USA).

Immunofluorescence experiments were performed on eyecup sections obtained from WT and Prpf31 $1^{A 216 P /+}$ mice. Serial sections of $18 \mu \mathrm{m}$ thick were mounted in five parallel series and processed for immunofluorescence. After 4\% PFA fixation and cryopreservation, retinal sections were incubated overnight at $4{ }^{\circ} \mathrm{C}$ with the primary antibodies: goat anti-PRPF31 (1:100; OriGene Technologies Inc., TA302582), mouse anti-RPE65 (1: 100; Abcam, ab78036), rabbit anti-Laminin (1:250; Sigma-Aldrich, L9393), mouse anti-HSPA4L (1:100; Santa Cruz Biotechnology, California, USA, SC-137007) and rabbit anti-HSP27 (1:1000 Enzo Life Sciences, New York, USA, ADI-SPA-803). After incubation, samples were washed 3 times in $0.2 \%$ PBS- $\mathrm{T}$, and incubated with appropriate AlexaFluor secondary antibodies (Molecular Probes, Oregon, USA) at room temperature for $1 \mathrm{~h}$. After 3 washes, sections were mounted with Vectashield mounting medium containing DAPI (Vector Laboratories). Sections of all analysed cases were processed in parallel following an identical protocol without the incubation step with the primary antibody, to be used as controls for immunoreaction specificity. To detect cholesterol accumulation, retinal sections were incubated with Filipin III (Sigma-Aldrich) for $2 \mathrm{~h}$ at room temperature. Whole mount of the RPE was performed as usually, and F-actin was stained with TRITCphalloidin (Sigma-Aldrich) according to the manufacturer's instructions.

Immunofluorescence experiments were also performed in cells grown on glass coverslips. Cells were fixed in $4 \%$ PFA and then permeabilized and blocked with $2 \%$ donkey serum/PBS-T for $1 \mathrm{~h}$ at room temperature.
Incubation with primary antibodies: goat anti-PRPF31 (1:100; OriGene Technologies Inc., TA302582) and mouse anti-HSP70 (1:100; Santa Cruz Biotechnology, SC-24) was performed for $1 \mathrm{~h}$ at room temperature. Cells were washed three times with PBS-T and incubated with AlexaFluor secondary antibodies (Molecular Probes). Coverslips were mounted on glass slides with Vectashield mounting medium containing DAPI (Vector Laboratories). Confocal images of retinal sections and cell coverslips were captured by a spectral confocal microscope TCS SP5 (Leica, Wetzlar, Germany) with an HCX PL APO Lambda blue 63 1.4. OIL objective, at $22^{\circ} \mathrm{C}$. MetaMorph Microscopy Automation and Image Analysis Software were used to analyse the images, and quantification of colocalization signal was obtained using Mander's overlap coefficient. Adobe Photoshop CS5.1 software was used for digital amplification of the images.

\section{Lipofuscin staining}

Retinal sections were incubated with carbol-fuchsine solution (4 $\mathrm{g}$ of fuchsine; $8 \mathrm{~g}$ of phenol, $20 \mathrm{~mL}$ of absolute ethanol and $100 \mathrm{~mL}$ of distilled water), for $1 \mathrm{~h}$ at room temperature. After 3 washes with distilled water, slides were cleared with alcohol acid solution (1\% hydrochloric acid in $70 \%$ ethanol). The slides were then washed with tap water for $5 \mathrm{~min}$ and counterstained with $2 \%$ picric acid. Finally, slides were dehydrated with rising alcohol solutions and cleared with xylene.

\section{Transmission electron microscopy (TEM) images}

Mice were anesthetized by subcutaneous injection of ketamine hydrochloride/xylazine solution $(80 / 12 \mathrm{mg} / \mathrm{kg}$ body weight) and perfused using a fixation solution containing $2.5 \%$ of PFA and $2.5 \%$ of glutaraldehyde in PBS. Eyes were enucleated and fixed overnight at $4{ }^{\circ} \mathrm{C}$ in the same fixation solution. TEM was performed by Nanoimaging Service in BIONAND (Malaga, Spain), using a FEI Tecnai G2 29 TWIN Transmission Electron Microscope.

\section{Dissection of mouse neuroretina and RPE for protein and mRNA extractions}

The animals were euthanized by cervical dislocation and the eyes quickly excised. The cornea was cut towards corneal limbus, using a small spring scissor. Then, the back of the eye was gently pressed to remove the lens. Four cuts were made perpendicular to the corneal limbus and towards the optic nerve head. The eye was opened in four petals, and finally the neuroretina was carefully separated from the underlying RPE-choroid using curved forceps. Samples were collected in separate microcentrifuge tubes for subsequent protein or mRNA extraction. 


\section{Western blot}

Proteins were extracted in ice-cold RIPA buffer containing protease inhibitor cocktail. Soluble/insoluble fractioning was performed as previously described (Diaz-Corrales et al., 2005). Briefly, cell lysates were incubated on ice for $60 \mathrm{~min}$ and the homogenates were centrifuged $(19,200 \times g$, $20 \mathrm{~min}$ at $4{ }^{\circ} \mathrm{C}$ ). The supernatants (detergent-soluble fraction) were collected and the pellets (detergent-insoluble fraction) were re-suspended in resuspension buffer (60 $\mathrm{mM}$ Tris- $\mathrm{HCl}$, 2\% SDS, 2.5\% 2-mercaptoethanol) and sonicated for $20 \mathrm{~min}$, at $4{ }^{\circ} \mathrm{C}$. Nuclear and cytosolic fractions were collected using the Ne-Per Nuclear and Cytoplasmic extraction reagents (Thermo Fisher Scientific). Protein content was measured by $D C^{\mathrm{max}}$ protein assay (Bio-Rad, California, USA) and samples stored at $-80^{\circ} \mathrm{C}$. Thirty micrograms of each extract were separated in a denaturing $10 \%$ SDS-PAGE gel and the proteins transferred to a PVDF membrane (Amersham Biosciences, Little Chalfont, UK), and blocked using Superblock Blocking buffer (Thermo Fisher Scientific, Massachusetts, USA) containing $0.1 \%$ of Tween-20 (Sigma-Aldrich) for $1 \mathrm{~h}$ at room temperature. The primary antibodies: anti-PRF31 (1:3000, Santa Cruz Biotechnology, SC-68347), mouse antiRhodopsin, (1:1000, Abcam, ab190307), mouse antiRPE65, (1:5000, Abcam, ab78036), mouse anti-HSPA4L (1:500, Santa Cruz Biotechnology, SC-137007), mouse anti-HSP70 (1:2000, Santa Cruz Biotechnology, SC-24), mouse anti-GAPDH (1:1000, Abcam, ab9484) and mouse anti- $\gamma$-Tubulin (1:2000, Sigma-Aldrich, T-5192) were incubated overnight at $4{ }^{\circ} \mathrm{C}$. The primary antibody mouse anti-FLAG ${ }^{\circ}$ M2 (1:1500, Sigma, F3165) was incubated for $1 \mathrm{~h}$ at room temperature. The membrane was probed with the appropriate anti-HRP-conjugated secondary antibodies for $1 \mathrm{~h}$ at room temperature, and the immunereactive bands were detected by chemiluminescence using ECL plus (Amersham Biosciences). Immunoreactive bands were quantified by densitometric analysis using ImageJ software, and normalized with GAPDH or $\gamma$ tubulin immunoreactive bands.

\section{Microarrays for gene expression analysis and alternative splicing}

Eight-month old WT and Prpf $31^{A 216 P /+}$ mice were sacrificed by cervical dislocation and total RNA from RPE was extracted using High Pure RNA tissue kit (Roche, Mannheim, Germany), according to manufacturer's instructions. Quality of isolated RNA was evaluated by RNA 6000 Nano assay on a 2100 Bioanalyzer (Agilent Technologies, California, USA). The RNA extracted from RPE/Choroid samples (100 ng) was used to produce end-labeled biotinylated ssDNA. The labeled ssDNA was hybridized using oligonucleotide microarray GeneChip ${ }^{\odot}$ MTA 1.0 (Affymetrix, California, USA), according to manufacturer's instructions. The arrays were scanned using the GeneChip ${ }^{\oplus}$ Scanner $30007 \mathrm{G}$ (Affymetrix) and analyzed with the GeneChip ${ }^{\circ}$ Command Console Software (Affymetrix). The raw array data were preprocessed and normalized using the Signal Space Transformation-SST Robust Microarray Analysis-RMA (Irizarry et al., 2003). Genes differentially expressed (fold change linear $<-2$ or $>2$ and ANOVA $p$-value $<0.05$ ) were selected for further analysis. Gene ontology was evaluated through the Database for Annotation, Visualization and Integrated Discovery (DAVID) v6.8 (Sherman \& Lempicki, 2009). For alternative splicing analysis, the data was normalized by Robust Multiarray Average-RMA and applying Detection Above the Background-DABG method. The splicing index was determined to evaluate the difference of expression of a given exon between $\operatorname{Prpf} 31^{A 216 P /+}$ and WT mice, excluding the influence of gene level expression. Exons differentially expressed (splicing index $=$ fold change linear < -2 or $>2$ and ANOVA $p$-value $<0.05)$ were selected for further analysis.

\section{RT-PCR and quantitative RT-PCR (qPCR)}

Total RNA from neuroretina and RPE samples was extracted using High Pure RNA tissue kit (Roche) according to the manufacturer's instructions. After spectrophotometric quantification of RNA using NanoDrop1000 (Thermo Fisher Scientific), reverse transcription was carried out using cDNAQuantiTect ${ }^{\oplus}$ reverse transcription kit (Qiagen, Hilden, Germany), according to manufacturer's instructions. cDNA amplification was performed by using $1 \mu \mathrm{g}$ of RNA as template. Approximately $100 \mathrm{ng}$ of cDNA was used for qPCR. Specific primers for Prpf31 (Mm01329809_m1, Thermo Fisher Scientific), Recoverin (Mm00501325_m1, Thermo Fisher Scientific), Rpe65 (Mm00504133_m1, Thermo Fisher Scientific) and Hspa4l (Mm00495441_m1, Thermo Fisher Scientific) were used. The qPCR was performed using $\operatorname{TaqMan}^{\circ}$ Gene Expression Real Time qPCR assays (Life-Technologies, California, USA) according to the manufacturer's instructions, using a Thermal Cycler C100 (Bio-Rad). The average cycle threshold (CT) of fluorescence units was used to analyze the mRNA levels. Prpf31, Recoverin, Rpe65 and Hspa4l mRNA levels were normalized by Gapdh RNA levels. Quantification was calculated as: mRNA levels (percent of control) $=2^{\Delta(\mathrm{CT})}$ with $\Delta(\mathrm{CT})=\mathrm{CT}_{(\text {Prpf31/Recoverin } / \text { Rpe65/Hspa4l })}-\mathrm{CT}_{(\text {Gapdh })}$.

\section{Funduscopy}

Mouse retinas were evaluated in vivo using an advanced retinal-imaging microscope (MICRON III, Phoenix Research Laboratories, Inc., California, USA). The animals were anaesthetized by subcutaneous injection of ketamine hydrochloride/xylazine solution $(80 / 12 \mathrm{mg} / \mathrm{kg}$ body weight) and pupils were dilated with one drop of $10 \%$ 
phenylephrine and 1\% tropicamide. Additionally, eyes were locally anaesthetized with $0.1 \%$ tetracaine and $0.4 \%$ oxybuprocaine and a generous amount of $1 \%$ methylcellulose was placed on the mouse corneas to keep the eye moist. Correct alignment of the eye and dilatation of the pupils were checked before placing the camera lens in contact with the cornea to visualize the retina. Finally, images of the central and the peripheral regions of the retina were repeatedly captured with a three-separate charge-coupled device camera. A short wavelength excitation filter (486.5 nm transmission band Tavg N 90\% 451.5) and a long wavelength emission filter (transmission band Tavg N 93\% 504.7-900 nm) were used to detect autofluorescence signal.

\section{ERG recordings}

ERG is used to measure the electrical response of retinal cells (photoreceptors, RPE cells, etc) to light stimuli. Whole field ERG was recorded in a Ganzfeld Color Dome (Diagnosys LCC). To assess scotopic vision, mice were dark adapted overnight. Anesthesia and pupil dilation of mice were performed as described above. A ring electrode made of gold wire (active electrode) was placed on the surface of the cornea which was previously treated with a wetting agent ( $1 \%$ methyl cellulose). Needle electrodes made of stainless steel were used as reference (forehead) and ground electrodes (tail). The narrow band filter was adjusted to frequencies of 0.312 to 300 $\mathrm{Hz}$. A single flash white $(6500 \mathrm{~K})$ was used as stimulus divided in 6 stages of progressive intensity at $0.01,0.05$, $0.2,1,3$ and $10 \mathrm{~cd}(\mathrm{~cd}) . \mathrm{s} / \mathrm{m}^{2}$. Fifteen responses were recorded at each stage with an interval of $15 \mathrm{~s}$ between each stimulus. To evaluate photopic vision, mice were adapted to light for $10 \mathrm{~min}$ with a background illumination of $30 \mathrm{~cd} / \mathrm{m}^{2}$. The intensity of the stimulus was 3,5 , 10,15 and $20 \mathrm{~cd} . \mathrm{s} / \mathrm{m}^{2}$. The amplitude and frequency of $\mathrm{a}$ - and b-waves were evaluated. To measure the c-wave the narrow and broad band filters were adjusted to 0.1 $\mathrm{Hz}$ and $30 \mathrm{~Hz}$, respectively. The c-wave value was measured at the maximum peak of the c-wave. A single green flash of $64 \mathrm{~cd} / \mathrm{m}^{2}$ during $200 \mathrm{~ms}$ was used was used as stimulius and the recording was extended until $4 \mathrm{~s}$.

\section{Plasmids}

pEGFP-N1 (Clontech, Michigan, USA) containing the CMV promoter was used as backbone. Human PRPF31 was amplified with specific primers containing NheI (5') and BamHI (3') restriction sites. PRPF31 $1^{A 216 P}$ was obtained by using a GeneArt ${ }^{\oplus}$ Site-Directed Mutagenesis System kit (Invitrogen, California, USA). The amplified fragments were cloned in pEGFP-N1. The resulting constructs (PRPF31-GFP and A216P-GFP) were confirmed by restriction enzyme digestion and sequencing. The
pcDNA3.1-PRPF31-C(K) DYK plasmid (PRPF31-Flag) was acquired from GenScript (New Jersey, USA).

\section{Cell culture}

Human RPE cell line ARPE-19 (ATCC ${ }^{\circ}$ CRL-2302 $2^{\text {ma }}$ ) was kept in culture at $37^{\circ} \mathrm{C}$ in a humid chamber with $5 \%$ $\mathrm{CO}_{2}$ and grown in Dulbecco's modified Eagle's medium F12 (DMEM/F12; Sigma-Aldrich) supplemented with 1\% penicillin/streptomycin (Sigma-Aldrich), 1\% glutamine (Sigma-Aldrich), and 10\% fetal bovine serum (Sigma-Aldrich). Culture medium was changed every 2 days. Transfection was performed using Lipofectamine 2000

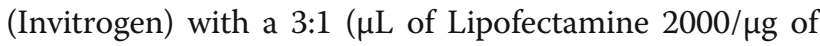
DNA) ratio, according to manufacturer's instructions. Briefly, $7.5 \times 10^{5}$ cells were seeded in a 6 -cm culture dish (Orange Scientific, Belgium), and $24 \mathrm{~h}$ after seeding, cells were transfected with $1 \mu \mathrm{g}$ of DNA. Twenty-four-hour post-transfection, cells were either fixed or collected for protein isolation, depending on the experiment. Cells were also transfected with the PRPF31-GFP or A216PGFP plasmids alone and co-transfected with the PRPF31-Flag plasmid.

\section{Fluorescence recovery after bleaching (FRAP) assay}

ARPE-19 cells transfected either with PRPF31-GFP or A216P-GFP constructs were used for FRAP experiments. FRAP assay was performed using a laser scanning confocal microscope TCS SP5 (Leica) equipped with an environmental control system for temperature $\left(37^{\circ} \mathrm{C}\right)$, humidity and $\mathrm{CO}_{2}$ concentration (5\%). Briefly, transfected cells from each condition were selected and imaged before bleaching. Photo-bleaching was applied in a circular region of interest-ROI of the same diameter positioned in the cell nucleus of the selected cells using an Argon laser $(488 \mathrm{~nm})$. Pre-bleached images were recorded for $3 \mathrm{~s}(1 \mathrm{~s} /$ frame $)$ and the selected area was bleached for $1 \mathrm{~s}$ with a pulse of 488-nm laser, at maximum intensity. After bleaching, a series of images were captured every second for $200 \mathrm{~s}$. Normalization was performed using the values before bleaching and the first time point after bleaching.

\section{Statistical analysis}

The SSPS software was used for statistical analysis. All experimental measurements were expressed as the means \pm SEM or quartiles in boxplot diagrams. Normal distribution of samples was evaluated by Kolmogorov-Smirnov. The samples of both eyes were pooled in one sample for protein and mRNA extractions. Statistically significant differences between groups were estimated by $t$-test, one-way ANOVA or the nonparametric Mann-Whitney $U$-test. A $p$ value $<$ 0.05 was considered statistically significant. 


\section{Results \\ Prpf31 ${ }^{\mathrm{A} 216 \mathrm{P} /+} \mathrm{KI}$ mice display RPE degeneration with drusen-like deposits}

To better understand the role of Prpf31 in retinal degeneration, we have used heterozygous Prpf31 $1^{A 216 P /+}$ KI mice (Bujakowska et al., 2009), a mouse model which carries the point-mutation p.A216P in the Prpf31 gene, known to be responsible for adRP in humans (Vithana et al., 2001). However, in mice p.A216P does not produce a photoreceptor cell death phenotype as it does in humans. On the other hand, it is known that Prpf $31^{+/-}$ KO mice display an RPE-degenerative phenotype. For this reason, we decided to characterize, in detail, the RPE-degenerative phenotype of aged KI mice. We started by studying funduscopy images in 8 to 16month-old mutant mice and their WT-littermates to evaluate the appearance of the retina. Ophthalmoscopic images of the central and peripheral regions of the retina showed normal appearance in the WT mice (Fig. 1a-d). The homogeneity in the surface of the central (Fig. 1a) and peripheral (Fig. 1b) retina is clearly shown, as well as the normal size of the optic nerve head (Fig. 1c) and the normal thickness of the blood vessels (Fig. 1d; white arrowhead). In contrast, small round, white-yellowish, non-confluent, scattered lesions were observed throughout the retina of $\operatorname{Prpf} 31^{\mathrm{A} 216 \mathrm{P} /+}$ mice like drusenoid deposits (Fig. 1e-h). Most of these lesions were autofluorescent (Fig. 1i-j; white arrows). The number of drusen-like deposits begins to be observed since the 8th month, and their number progressively increases in a non-homogeneous way during the retinal degenerative process. In the mutant mice evaluated we did not observe any of the typical fundus features of RP, such as black pigment accumulation in the form of bone spicules, vascular sharpening or optic nerve head atrophy.

TEM of 8-month-old WT (Fig. 1k, l) and KI retinas (Fig. 1m-o) was also performed to evaluate the morphology of RPE cells and the Bruch's membrane in detail. In the WT mice, the normal expected morphology for RPE was observed, with the presence of photoreceptor outer segments (OS) in contact with RPE apical microvilli (Mv) (Fig. 1k; OS and Additional file 1: Figure S1a), melanin and lipofuscin granules in the cytoplasm (Fig. 1k; Me, Lf) as well as basal infoldings of the RPE membrane (Fig. $1 \mathrm{k} ; \mathrm{BI}$ ) in contact with the Bruch's membrane (Fig. $1 \mathrm{k}$; BM). Bruch's membrane presented a welldefined fundamental structure (Fig. 1l; fs) between each basal lamina, one corresponding to the RPE and the other to the endothelium of a choroidal vessel (Fig. 1l; BL, arrowheads). The thickness of the Bruch's membrane measured between both basal laminae was $400 \mathrm{~nm}$ (Fig. 1 l). In contrast, Prpf31 $1^{\mathrm{A} 216 \mathrm{P} /+}$ TEM images showed, accumulation of lipofuscin granules (Fig. 1m, o; Lf), vacuolization of the RPE (Fig. 1m; Va), atrophy of basal infoldings (Fig. 1m;
BI) and thickening of Bruch's membrane (Fig. $1 \mathrm{~m}$; BM), to an approximate size of $700 \mathrm{~nm}$ (Fig. 1n). Additionally, the homogeneity of the fundamental membrane structure was lost (Fig. 1n; fs) and we also found accumulation of amorphous electrodense material within the Bruch's membrane (Fig. 1o; black arrow). The Mv and the end of OS in mutant mice were also observed. Mv were shorter and disorganized when compared with the Mv of WT mice (Additional file 1: Figure S1). Despite all these alterations, the $\operatorname{Prpf} 31^{A 216 P /+}$ mice did not show any damage of the photoreceptor.

In addition, specific staining methods were used to visualize lipofuscin granules (Fig. 1p, r). Large accumulation of lipofuscin granules was observed in the RPE of the mutant mice (Fig. 1r; dark magenta) compared to WT (Fig. 1p). To evaluate the composition and localization of the amorphous material observed in the Bruch's membrane, Filipin blue staining was used to detect free cholesterol (Fig. 1q, s, t; blue). Immunofluorescence for laminin (Fig. 1 q, s, t; red) and Rpe65 (Fig. 1q, $\mathrm{s}$, $\mathrm{t}$; green) are shown as markers of basal lamina and $\mathrm{RPE}$, respectively. In the KI mice, accumulation of free cholesterol was observed between the RPE and the Bruch's membrane (Fig. 1s) or between both basal laminae (Fig. 1t). Localization of these deposits is similar to the basal linear deposits and basal lamellar deposits described by Curcio and colleague in age-related macular degeneration (AMD) (Curcio \& Millican, 1999). RPE atrophy, lipofuscin accumulation and thickening of Bruch's membrane are also features described in AMD (Curcio \& Millican, 1999; Ding et al., 2009).

Finally, we have monitored ERG responses in the KI mice and found that a- and b-waves, corresponding to photoreceptor electrical activity, were not affected (Additional file 1: Figure S2a-c"). This is similar to what was previously reported for all splicing factor-mutant mouse models (Bujakowska et al., 2009; Farkas et al., 2014). Surprisingly, a defective c-wave was observed, reflecting, at a functional level, the specific degenerative changes found in the RPE layer (Additional file 1: Figure S2d-d"). Therefore, the Prpf $31^{A 216 P /+}$ mice display RPE degeneration with drusen-like deposits.

\section{PRPF31 is highly expressed in the RPE}

We have analysed expression of Prpf31 in retinal samples of WT CD-1 mice to examine its distribution in the different retinal layers. Immunohistochemistry (Fig. 2) shows Prpf31 to be highly expressed in the RPE cell layer (Fig. 2a; arrowheads) compared to neuroretina, where almost no Prpf31 signal was detected. Rhodopsin, the photo-pigment expressed in rod-photoreceptors, was used as a positive control for the immunohistochemical staining (Fig. 2b) and, as expected, a clear signal was displayed in the photoreceptor OS. A negative control, 


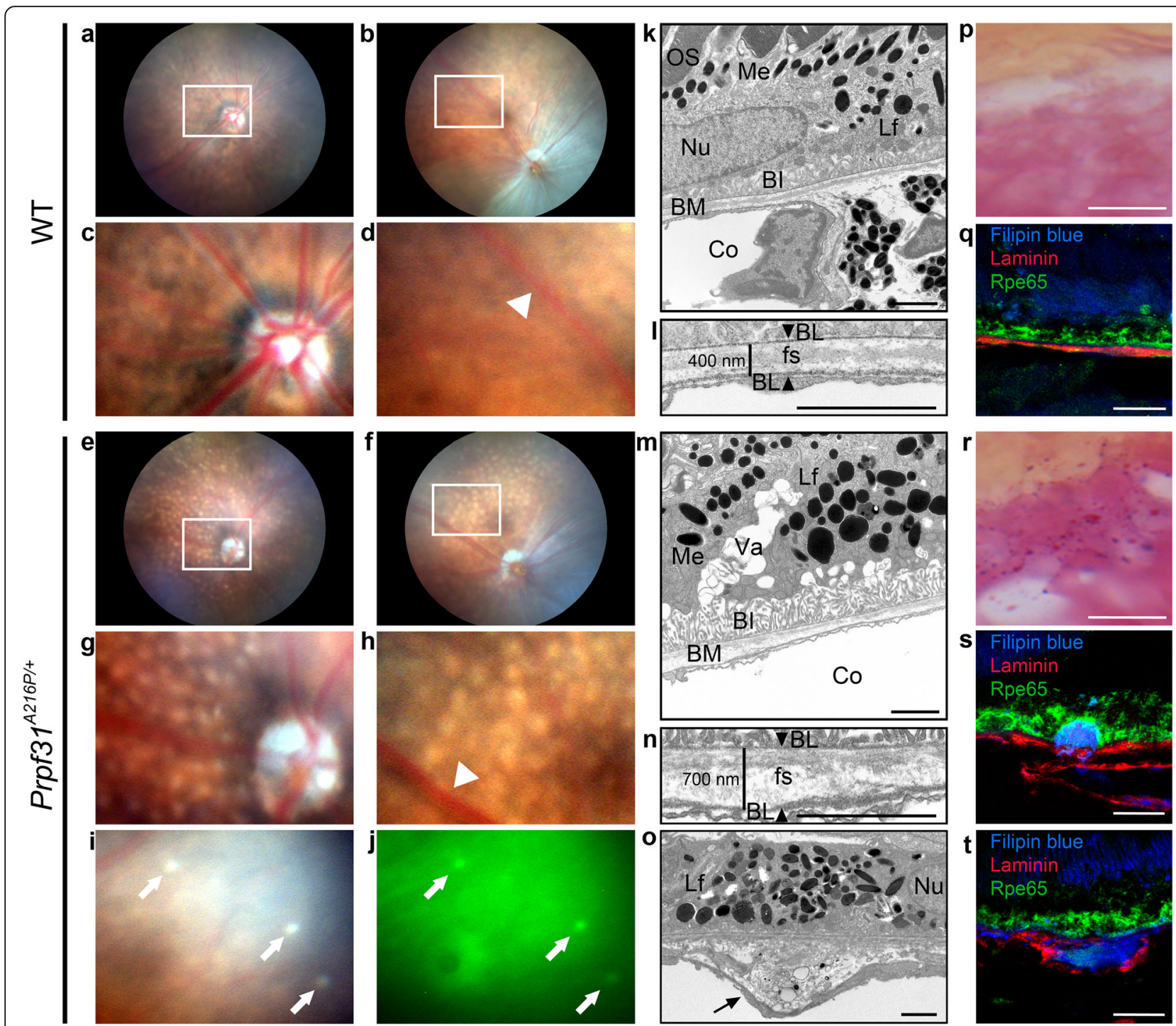

Fig. 1 Prpf3 $1^{A 216 P /+}$ mice exhibit degenerative phenotype of the RPE with drusen-like deposits. Funduscopy of WT (a-d) and Prpf31 ${ }^{A 216 P /+}$ mice (ej) are shown. Numerous white-yellowish round lesions were observed in the retina of Prpf3 $1^{A 216 P /+}$ mice (e-h). These lesions were distributed in the central $(\mathbf{g})$ and peripheral retina $(\mathbf{h})$ and most of them showed autofluorescence (i, j; white arrows). The optic nerve head $(\mathbf{g})$ and retinal vessels (h; white arrowhead) did not show differences when compared to WT mice (c, $\mathbf{d}$; white arrowhead). TEM images of RPE of 8-month-old WT $(\mathbf{k}, \mathbf{l})$ and Prpf3 $1^{A 216 P /+}$ mice $(\mathbf{m}-\mathbf{o})$ and the amplified images of Bruch's membrane (BM) are displayed $(\mathbf{I}, \mathbf{n}, \mathbf{o})$. Photoreceptor outer segments (OS) were observed in contact with the RPE microvilli in WT mice (k). Accumulation of lipofuscin granules (Lf), large vacuoles (Va) and atrophy of basal infoldings (BI) were observed in the RPE of Prpf3 $1^{A 216 P /+}$ mice $(\mathbf{m})$. The distance between both basal laminae (BL) was measured $(\mathbf{I}, \mathbf{n}$; arrowheads), and thickening of the BM was detected in Prpf3 $7^{A 216 P /+}$ mice (n). In addition, the homogeneity of the fundamental substance (fs) was lost and amorphous electrodense material was accumulated within the BM of these mice (o; black arrow). The morphology of melanin granules (Me), nuclei (Nu) and choroid (Co) was normal. Lipofuscin staining in dark magenta ( $\mathbf{p}, \mathbf{r})$ showed large accumulation of lipofuscin granules in the RPE of Prpf3 $7^{A 216 P /+}$ mice (r). Filipin blue dye was used to stain free cholesterol (q, $\mathbf{s}, \mathbf{t} ;$ blue). Prpf3 ${ }^{A 216 P /+}$ mice showed free cholesterol accumulation (s, t: blue) between the RPE and BM (s) or within the BM (t). Anti-Laminin antibodies were used to stain the BL $(\mathbf{q}, \mathbf{s}, \mathbf{t}$; red) and the RPE was visualized by anti-Rpe65 antibody (q,s, t; green). Scale bars represent $2 \mu \mathrm{m}$ (k-o) or $12.5 \mu \mathrm{m}$ (p-t)

without primary antibody, was performed to discard non-specific binding of secondary antibody (Fig. 2c).

To compare differential expression of Prpf31 protein in different layers of the mouse retina, neuroretina and RPE were manually dissected and protein and mRNA samples were obtained from each fraction. Immunoblotting results indicate high expression of $\operatorname{Prpf} 31$ protein compared to the neuroretina (Fig. 2d). Antibodies against Rhodopsin and Rpe65, an enzyme of the visual cycle cascade expressed in the RPE, were used as fraction-specific markers (Fig. 2d). This result was further confirmed by qPCR (Fig. 2e), in which it is possible to observe that Prpf31 expression level is much higher in the RPE, when compared to its expression in the neuroretina. Recoverin 


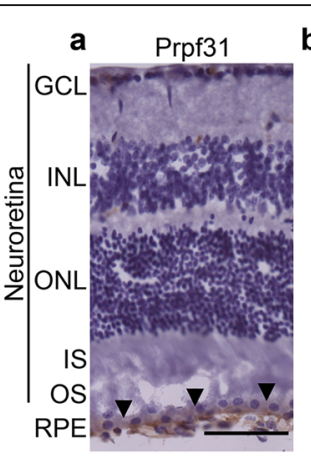

e

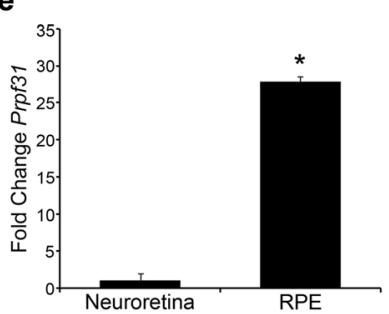

b Rhodopsin

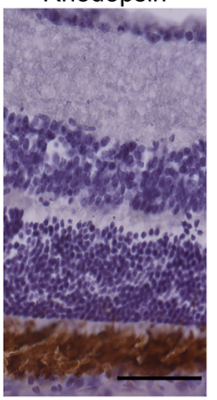

f

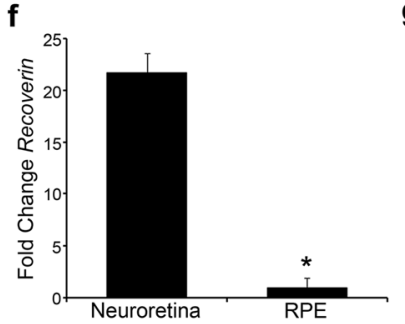

d
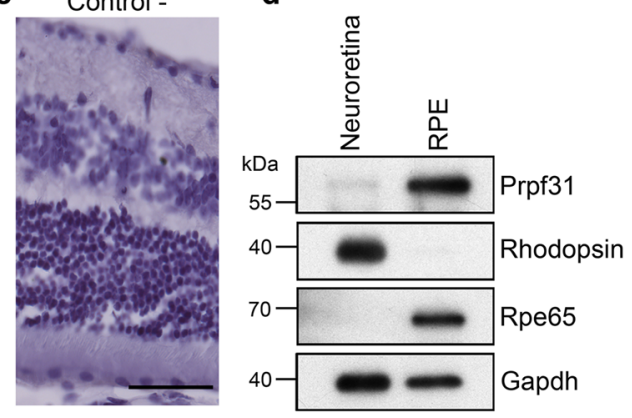

g

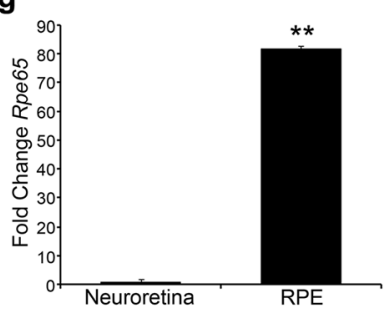

Fig. 2 Prpf31 protein and its mRNA are highly expressed in the RPE of mouse retinas. Immunohistochemical staining showed strong Prpf31 signal in the RPE of CD-1 mouse retinas (a; arrowheads). Anti-Rhodopsin antibodies were used as positive control for immunohistochemical staining (b). A negative control without primary antibodies is also present (c). Western blot (d) and GPCR (e) analysis of Prpf31 protein and mRNA expression in the neuroretina and RPE samples showed that it is mainly expressed in the RPE (d, e). Anti-Rhodopsin and anti-Rpe65 antibodies were used as controls for the neuroretina/RPE tissue fractions, and anti-Gapdh antibody was used as a loading control (d). For qPCR, Recoverin (f) and Rpe65 (g) mRNA expression levels were used as controls for the two different tissue fractions. The bars in the graphs $\mathbf{e}-\mathbf{g}$ represent means of fold change \pm SEM ( $n=4$ replicates of 3 samples in each group). Statistically significant differences were determined by $t$-test $\left({ }^{*} p<0.05,{ }^{*} p<0.01\right)$. RPE $=$ retinal pigment epithelium, OS = outer segment, IS = inner segment, $\mathrm{ONL}=$ outer nuclear layer, $\mathrm{INL}=$ inner nuclear layer, $\mathrm{GCL}=$ ganglion cell layer. Scale bars represent $50 \mu \mathrm{m}$

and Rpe65 mRNAs were used as markers for neuroretina and RPE fractions, respectively (Fig. $2 \mathrm{f}-\mathrm{g}$ ).

To confirm whether this differential distribution of PRPF31 along the retinal cell types is common to other vertebrates, fractions of RPE and neuroretina were obtained from C57BL/6 J mice, pig, cow and human eye samples. The immunoblot for PRPF31 (Additional file 1: Figure S3) showed that protein level is comparatively higher in the RPE than in the rest of the retinal layers in several vertebrates, including humans.

\section{Mutant PRPF31 protein is aggregated in the cytoplasm of RPE cells}

Next, we were interested in the distribution of the Prpf31 protein histologically in the RPE of the aged-KI mice. Immunofluorescence analysis of $\operatorname{Prpf} 31$ protein did not show clear differences between WT and KI mice in retinal sagittal sections. However, RPE whole-mount evaluation showed large cytoplasmic aggregates of Prpf31 protein in $\operatorname{Prpf} 31^{A 216 P /+}$, which were almost not present in the WT samples (Fig. 3e-h). Additionally, a difference in the Prpf31 distribution within the RPE cell was observed, with a weaker staining in the KI nuclei compared to the WT-littermates (Fig. 3e-h). In the WT RPE cells, most of Prpf31 is localized inside the nucleus with some homogeneous cytoplasmic staining (Fig. 3a-d) but in the Prpf $31^{A 216 P /+}$ RPE cells, most of the Prpf31 staining is shown in the cytoplasm forming rounded clumps of Prpf31 protein resembling aggregates (Fig. 3e-h). Cells that were observed harbouring protein aggregates formed clusters in the RPE layer of the KI mice. The number of cells with cytoplasmic aggregates was counted in both KI and WT mice, providing a statistically significant difference, with $\operatorname{Prpf} 31^{A 216 P /+}$ mice having $18.4 \pm 2.3 \%$ of RPE cells with cytosolic aggregates compared to $1.7 \pm 0.2 \%$ cells in the WT mice (Fig. 3i).

To quantify the amount of Prpf31 signal in the RPE nuclei, the Manders' overlap coefficient for DAPI+Prpf31/DAPI colocalization (M1) and for Prpf31 + DAPI/Prpf31 colocalization (M2) were calculated (Fig. 3j). Both coefficients were significantly lower in the mutant mice (Fig. 3j), corresponding to the diminished amount of Prpf 31 protein in the nucleus of RPE cells as observed by histology. Besides, Western blot of cytosolic and nuclear fractions clearly showed a decrease of Prpf31 protein in the nuclear fractions of mutant mice (Fig. 3k). Therefore, these results show that not only Prpf31 protein in the $\operatorname{Prpf} 31^{A 216 P /+}$ mice is aggregated in the cytoplasm of the RPE cells, but also its concentration in the nuclei is decreased when compared to the WT. 


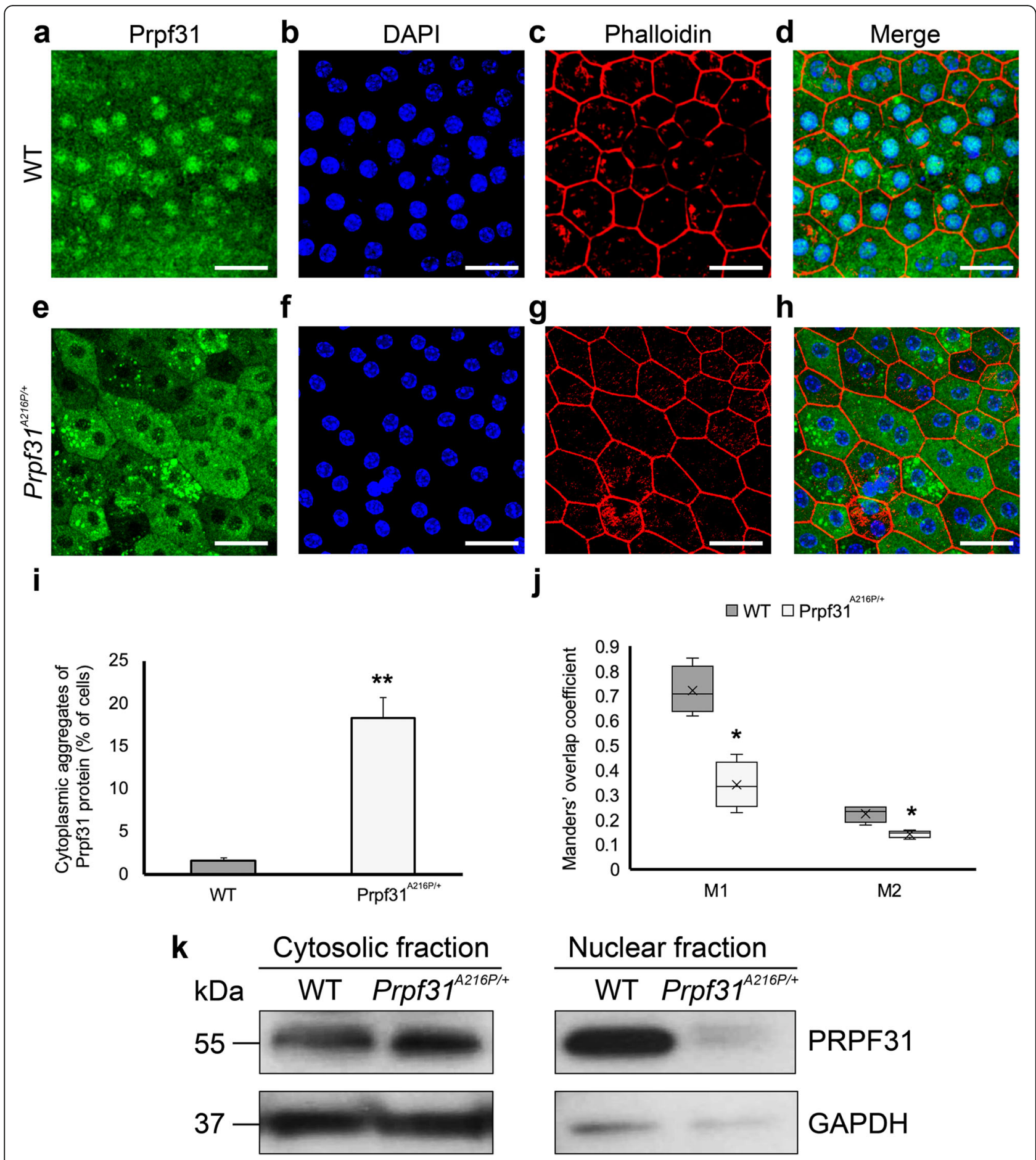

Fig. 3 Large cytoplasmic aggregates of Prpf31 protein were observed in the RPE of Prpf31 $1^{A 216 P /+}$ mice. Whole-mount of the RPE layer from WT (ad) and Prpf3 $1^{\text {A216P/+ }}$ mice (e-h) were immunostained with anti-Prpf31 antibodies (a, e). Cell nuclei were stained with DAPI (b, f), and TRITCphalloidin was used to visualize the F-actin microfilaments $(\mathbf{c}, \mathbf{g})$. Prpf31 signal was mainly localized in the nuclei of WT RPE cells (a), while large protein aggregates stained for PRPF31 were observed in the cytoplasm of RPE cells of mutant Prpf3 $1^{A 216 P /+}$ mice (e). The bars in graph $\mathbf{i}$ represent the percentage of RPE cells with cytoplasmic aggregates of Prpf31 protein \pm SEM in WT and Prpf3 $1^{\text {A2 } 216 P /+}$ whole mount RPE samples $(n=1200$ cells were counted from 4 mice in each group). The boxplot $\mathbf{j}$ represents the Manders' overlap coefficient of DAPI+Prpf31/DAPI colocalization (M1) and Prpf31 + DAPI/Prpf31 colocalization (M2) in WT and Prpf3 $1^{A 216 P /+}$ whole-mount RPE samples ( $n=4$ in each group). Expression of Prpf31 protein was evaluated in the cytosolic and nuclear fractions by Western blot (k). Statistically significant differences were determined by $t$-test or Mann-Whitney U-test $\left({ }^{*} p<0.01,{ }^{* *} p<0.001\right)$. Scale bars represent $25 \mu \mathrm{m}$ 
The antibody used to visualize Prpf31 recognized both mutant and normal Prpf31 protein, thus we were unable to determine whether the aggregates are composed solely of the mutated protein or if the WT protein is also present in the aggregates.

\section{Differential gene expression and alternative splicing were affected in Prpf31 $1^{\mathrm{A} 216 \mathrm{P} / \mathrm{H}}$ mice}

Considering that the mutant Prpf31 protein is aggregated in the cytoplasm of RPE cells in Prpf $31^{A 216 P /+}$ mice, we decided to perform transcriptomic analysis using a GeneChip $^{\text {tm }}$ Mouse Transcriptome Array (MTA) 1.0 to evaluate differential gene expression in RPE samples of six $\operatorname{Prpf}_{31} 1^{A 216 P /+}$ and three WT-littermates. The number of genes evaluated was 65,956 and, from these, a total of $1033(1.6 \%)$ genes were differentially expressed in the $\operatorname{Prpf}_{31} 1^{A 216 P /+}$ mice. Most of these genes were upregulated (922, 89.3\%) and the rest were downregulated (Table 1; Additional file 2, Gene expression). The gene-level differential expression analysis is graphically displayed in the volcano plot (Fig. 4a). Each point on the plot represents the statistical result of a single gene. Horizontal axis represents fold change in $\log 2$ scale and vertical axis represents $p$-value in $\log 10$ scale. Threshold of fold change was either $<-2$ (Fig. 4a; blue) or $>2$ (Fig. 4 a; red) and ANOVA $p$ value $<0.05$. Gray dots correspond to the genes without statistically significant change. Hierarchical clustering of 1033 genes differentially expressed in $\operatorname{Prpf} 31^{A 216 P /+}$ vs WT mice is shown in Fig. 4b with an expression profile clearly different for WT and Prpf31 $1^{A 216 P /+}$ clusters (Fig. 4b).

Gene ontology was evaluated through the informatic tool DAVID v6.8 (Sherman \& Lempicki, 2009) using the list of 1033 genes which were differentially expressed in Prpf31 $1^{A 216 P /+}$ mice, showing that the largest group of genes (123 genes) affected by the mutation belongs to Protein Binding (Additional file 1: Table S1). This molecular function is defined as: interacting selectively and noncovalently with any protein or protein complex (GO: 0005515), including the subcategory chaperone binding (GO:0051087); a class of proteins that bind to nascent or unfolded polypeptides to ensure correct folding or transport. Because the most relevant change observed in the RPE of the mutant mice was the cytoplasmic aggregation of Prpf31 protein, we decided to look for candidate genes related to molecular chaperones involved in protein folding. We found that heat shock protein family A (Hsp70) member 4 like gene (Hspa4l), which encodes the chaperone heat shock $70 \mathrm{kDa}$ protein $4 \mathrm{~L}$ (Hspa4l), was upregulated in Prpf $31^{A 216 P /+}$ mice (fold change 2.26; $p$ value 0.009 ). Other chaperones involved in the unfolded protein response were not highlighted with a different gene expression level. Lists of candidate genes found to be differentially expressed which might also be involved in RPE degeneration are shown in Additional file 1: Table S2.

Alternative splicing analysis was also performed through the GeneChip ${ }^{\text {tw }}$ MTA 1.0 in the same RPE samples of six Prpf31 $1^{A 216 P /+}$ and three WT-littermates. A total of 65,770 genes were evaluated and $92.6 \%$ (60871) of these genes were expressed in both mice (Prpf31 ${ }^{A 216 P /+}$ and WT mice). From these 60,871 genes, 6700 (11\%) genes have, at least, one differentially expressed probe selection region or junction to indicate alternative splicing (Table 2; Additional file 3, Splicing). The scatter plot (Fig. 4c) displays the number of genes that are alternatively spliced in $\operatorname{Prpf} 31^{A 216 P /+}$ mice with lower $<-2$ (Fig. 4c; red) or higher $>2$ (Fig. 4 c; blue) splicing index, when compared to WT mice.

Functional categories of alternative spliced genes in the RPE of Prpf $31^{A 216 P /+}$ mice are listed in the Additional file 1: Table S3. We observed that several splicing factors, including Prpf31, present a different splicing index (Prpf31 splicing index - 2.33, $p$-value 0.04; Prpf18 splicing index -2.62 , $p$-value 0.01; Prpf39 splicing index $2.25, p$-value 0.03$)$. Apart from the aforementioned splicing factors, others genes of different pathways involved in retinal degeneration were also affected by the mutation, such as inflammation, oxidative stress, retinol metabolism (Abca4), ciliogenesis (Bbs1, Bbs4, Bbs5, Bbs7, $B b s 9$ ) and cellular apoptosis (Additional file 1: Table S4;

Table 1 Summary of gene level differential expression analysis in RPE samples in two different conditions (Prpf3 $1^{\text {A216P/+ }}$ vs WT mice). Default filter criteria, fold change $<-2$ or $>2$ and ANOVA $p$-value $<0.05$

\begin{tabular}{|c|c|c|c|c|}
\hline $\begin{array}{l}\text { Type of } \\
\text { genes }\end{array}$ & $\begin{array}{l}\text { Number of genes } \\
\text { evaluated }(\%)\end{array}$ & $\begin{array}{l}\text { Number of genes differentially } \\
\text { expressed (\%) }\end{array}$ & $\begin{array}{l}\text { Number of genes up- } \\
\text { regulated }(\%)\end{array}$ & $\begin{array}{l}\text { Number of genes down- } \\
\text { regulated (\%) }\end{array}$ \\
\hline Non-coding & $36,703(55.7)$ & $562(0.9)$ & $485(47.0)$ & $77(7.5)$ \\
\hline Complex & $15,644(23.7)$ & $280(0.4)$ & 264 (25.6) & $16(1.5)$ \\
\hline Coding & $10,692(16.2)$ & $151(0.2)$ & 134 (12.9) & $17(1.6)$ \\
\hline Pseudogene & $2711(4.1)$ & $40(0.1)$ & $39(3.8)$ & $1(0.1)$ \\
\hline Ribosomal & $155(0.2)$ & $0(0.0)$ & $0(0.0)$ & $0(0.0)$ \\
\hline Unassigned & $51(0.1)$ & $0(0.0)$ & $0(0.0)$ & $0(0.0)$ \\
\hline Total & $65,956(100.0)$ & 1033 (1.6) & $922(89.3)$ & $111(10.7)$ \\
\hline
\end{tabular}

MTA 1.0, genome version $\mathrm{mm} 10$ 


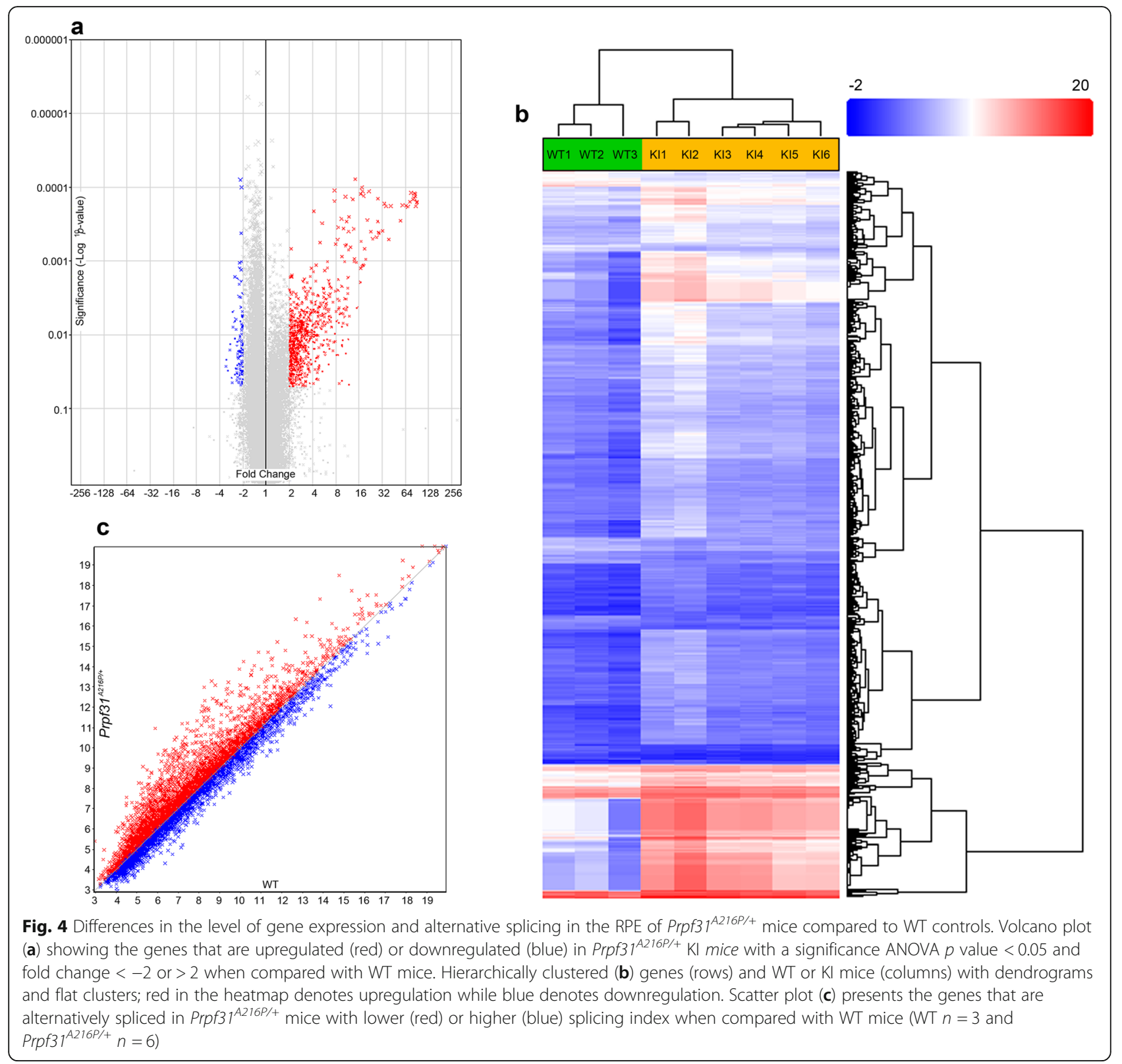

Table 2 Summary of alternative splicing analysis in RPE samples in two different conditions (Prpf3 $7^{\text {A216P/+ }}$ vs WT mice). Default filter criteria, splicing index $<-2$ or $>2$ and ANOVA $p$-value $<0.05$

\begin{tabular}{llll}
\hline $\begin{array}{l}\text { Type of } \\
\text { genes }\end{array}$ & $\begin{array}{l}\text { Number of genes } \\
\text { evaluated (\%) }\end{array}$ & $\begin{array}{l}\text { Number of genes expressed in both } \\
\text { conditions (\%) }\end{array}$ & $\begin{array}{l}\text { Genes with at least one differentially expressed PSR or junction to } \\
\text { indicate alternative splining (\%) }\end{array}$ \\
\hline Non-coding & $36,581(55.6)$ & $33,587(51.1)$ & $929(1.5)$ \\
Complex & $15,644(23.8)$ & $15,056(22.9)$ & $4344(7.1)$ \\
Coding & $10,628(16.2)$ & $9450(14.4)$ & $1381(2.3)$ \\
Pseudogene & $2711(4.1)$ & $2601(3.9)$ & $29(0.1)$ \\
Ribosomal & $155(0.2)$ & $155(0.3)$ & $16(0.0)$ \\
Unassigned & $51(0.1)$ & $22(0.0)$ & $1(0.0)$ \\
Total & $65,770(100.0)$ & $60,871(92.6)$ & $6700(11.0)$ \\
\hline
\end{tabular}


Additional file 3, Splicing). The number of candidate genes with modified splicing index that can be involved in RPE degeneration are detailed in Additional file 1: Table S4. These results suggest that normal splicing of different genes, including splicing factors, is affected in the RPE of Prpf $31^{A 216 P /+}$ mice.

\section{Increased Hspa4l expression and colocalization with mutant Prpf31 protein in the RPE of Prpf31 ${ }^{\mathrm{A} 216 \mathrm{P} /+}$ mice}

Analysis of transcriptomic data show that a member of the heat shock protein 70 (HSP70) family, Hspa4l, was overexpressed in the RPE of KI mice. The HSP70 family is a ubiquitous and conserved family of molecular chaperons assisting in protein folding to prevent aggregation and to protect cells from stress (Mashaghi et al., 2016; Mayer \& Bukau, 2005). We have analyzed mRNA expression of Hspa4l by qPCR in both RPE and neuroretina of the KI mice and we found that Hspa4l is overexpressed in the RPE of the mutant mice, when compared to its expression in the WT mice (Fig. 5a). No differences of Hspa4l expression in the neuroretina extracts were observed (Fig. 5a). This result was corroborated by Western blot in which we observed that Hspa4l was more abundant in the RPE of the mutant mice (Fig. 5b). RPE whole-mount immunofluorescence to localize Hspa4l and Prpf31 was also performed. As previously mentioned, Prpf31 protein predominantly localizes in RPE nuclei of WT samples (Fig. 5c, f; arrowhead) and in cytoplasmic aggregates in the case of KI tissue and low expression in the nucleus (Fig. 5i, l; arrow). As expected, Hspa4l staining is stronger in the mutant RPE cells, where the chaperone colocalizes with Prpf31 protein aggregates (Fig. 5i-n). The hexagonal shape of the RPE can be seen with phalloidin staining (Fig. 5c-n; blue). Negative controls for RPE autofluorescence and secondary antibodies nonspecific bindings are shown in the Additional file 1: Figure S4. The small heat shock protein, Hsp27, also colocalized with the Prpf31 aggregates in the RPE of mutant mice (Additional file 1: Figure S4), but transcriptomic data did not show differential expression of its gene ( $H s p b 1)$.

\section{The p.A216P mutant protein produces insoluble cytoplasmic aggregates, recruits endogenous PRPF31 protein in the insoluble fraction and increases the expression of HSP70}

To better understand the role of p.A216P mutation in human-derived RPE cells, we have cloned the human WT PRPF31 and the A216P mutant genes in pEGFP-N1 plasmid and the GFP-tagged PRPF31 proteins were overexpressed in ARPE-19 cells. We observed that WT PRPF31-GFP was mainly found in the nucleus of the transfected cells (Fig. 6a-c and g-i), while the mutant A216P-GFP was mostly aggregated in the cytoplasm of the transfected cells (Fig. 6d-f and j-l), similar to our previous observation in the RPE of the WT and Prpf3 $1^{A 216 P /+}$ mice, as shown in Fig. 3.

Western blot analysis of detergent-soluble and detergentinsoluble fractions of the cell lysates was conducted to estimate the concentration of PRPF31 in both fractions. Western blotting and densitometry analysis of the immunoblots (Fig. 6m, n) show less mutant protein A216P-GFP in the soluble fraction compared to PRPF31-GFP protein, and the opposite in the detergent-insoluble fraction (Fig. $6 \mathrm{~m}, \mathrm{n}$ ). Additionally, it is possible to observe a depletion of the endogenous PRPF31 protein in the soluble fraction and highest levels of expression in the insoluble fraction (Fig. $6 \mathrm{~m}$ ), suggesting that the cytoplasmic insoluble aggregates of $A 216 P$-GFP protein recruit the endogenous WT PRPF31 protein. To evaluate whether the WT PRPF31 protein increased in the insoluble fraction in cells cotransfected with the mutant protein PRPF31 (A216P), ARPE-19 cells were co-transfected with WT PRPF31 tagged to Flag (PRPF31-Flag) and PRPF31-GFP or A216PGFP. After $24 \mathrm{~h}$ of incubation, the protein extracts of soluble and insoluble fractions were analyzed by Western blotting (Fig. 6o). It was observed that the WT protein tagged to GFP was expressed in both the soluble and insoluble fraction, while the mutant protein was expressed mostly in the insoluble fraction (Fig. 6o). Besides, it was found that the WT protein tagged to Flag decreased in the soluble fraction and increased in the insoluble fraction only in those cells expressing the PRPF31 protein carrying the p.A216P mutation. In this way, we confirm our hypothesis that the mutant PRPF31 protein recruits the WT protein in the insoluble fraction.

HSP70 plays an important role in retinal dystrophies, including RP (Furukawa \& Koriyama, 2016) so we have also evaluated the distribution and expression of HSP70 protein in the ARPE-19 cells overexpressing either PRPF31-GFP or A216P-GFP by immunofluorescence and Western blotting. In the immunofluorescence staining, PRPF31-GFP transfected cells show a weak labeling of HSP70 in the nucleus (Fig. 7a-d). However, in A216PGFP transfected cells, HSP70 staining is increased, and its signal colocalizes with the cytoplasmic aggregates of mutant PRPF31 protein (Fig. 7e-h), similar to the distribution observed for Hspa4l in the RPE of Prpf31 ${ }^{A 216 P /+}$ mice. Additionally, Western blot analysis of the soluble and insoluble fractions (Fig. 7i) and its densitometry analysis (Fig. 7j) show an increase of HSP70 in the insoluble fraction of the cells transfected with A216P-GFP. Therefore, protein aggregation of mutant PRPF31 seems to activate the response of chaperones.

P.A216P mutation affects the mobility of PRPF31 protein to the nucleus in human RPE derived live cells

FRAP experiments provide qualitative and quantitative information about the mobility of a fluorescently tagged 


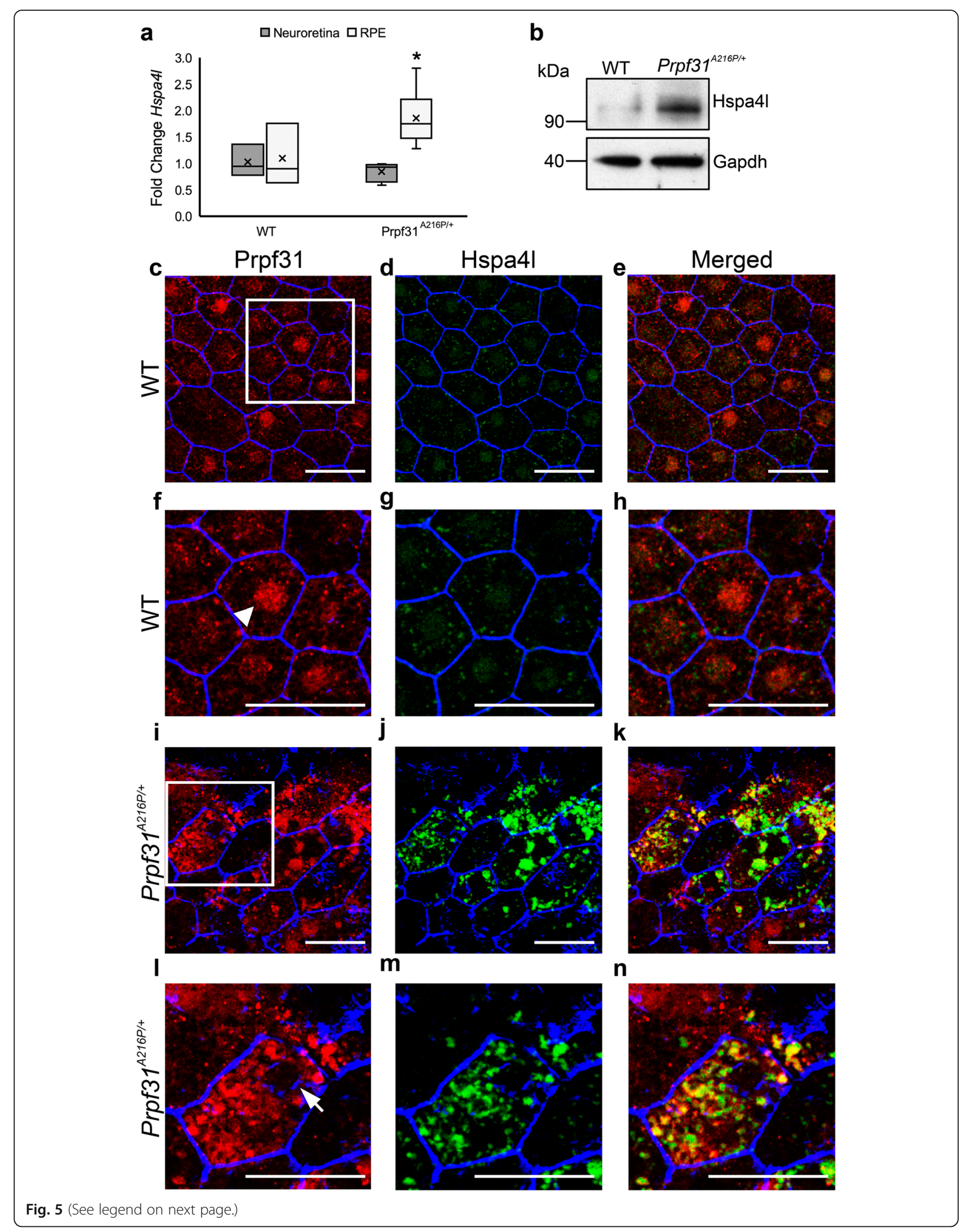


(See figure on previous page.)

Fig. $5 \mathrm{Hspa4l}$ is highly expressed in the RPE of Prpf3 $7^{A 216 P /+}$ mice. Analysis of Hspa4l expression by qPCR in the neuroretina and RPE samples show that Hspa4l mRNA is overexpressed in the RPE of Prpf3 $1^{A 216 P /+}$ mice (a). The boxplot a represents the fold change of Hspa4l expression in the neuroretina and RPE of WT and Prpf3 $1^{A 216 P /+}$ mice (WT $n=3$ and Prpf3 $\left.1^{A 216 P /+} n=6\right)$. Statistically significant differences were determined by Mann-Whitney $U$-test $\left({ }^{*} p<0.05\right)$. Western blot of RPE samples showed higher expression of Hspa4l protein in Prpf3 $1^{A 216 P /+}$ mice compared to WT (b). Anti-Gapdh antibodies were used as loading control (b). Whole-mount of the RPE obtained from WT (c-h) and Prpf3 $7^{A 216 P /+}$ mice (i-n) were immunostained with anti-Prpf31 (c, $\mathbf{f}, \mathbf{i}, \mathbf{l})$ and anti-Hspa4l antibodies $(\mathbf{d}, \mathbf{g}, \mathbf{j}, \mathbf{m})$. TRITC-phalloidin was used to stain F-actin microfilaments (c-n; blue). Magnified images are (f-h and $\mathbf{l}-\mathbf{n})$ and merged are shown $(\mathbf{e}, \mathbf{h}, \mathbf{k}, \mathbf{n})$ Prpf31 signal was mainly distributed in the nuclei of RPE cells in WT mice (c, $\mathbf{f}$; arrowhead), while Prpf31 protein aggregates were observed in the cytoplasm (i, I) colocalizing with Hspa4l signal in mutant Prpf3 $1^{\text {A216P/+ }}$ mice as shown in the merged images $(\mathbf{k}, \mathbf{n})$. Prpf31 signal was very low in the nuclei of Prpf3 $1^{\text {A216P/+ }}$ mice (l; arrow). Scale bars represent $25 \mu \mathrm{m}$

protein in a defined compartment (Phair \& Misteli, 2001; Reits \& Neefjes, 2001). Different parameters can be evaluated by FRAP, such as the mobile fraction $(\mathrm{mf})$ of a fluorescent-tagged protein and its half-life $\left(\tau_{1 / 2}\right)$. The $\mathrm{mf}$ can be affected by different circumstances, such as interaction of the fluorescent-tagged protein with other proteins, cytoplasmic organelles or membranes. Considering that p.A216P mutation induces protein aggregation and expression of HSP70, which colocalizes with the cytoplasmic aggregates of PRPF31 protein, we decided to investigate whether the translocation of PRPF31 protein from the cytoplasm to the nucleus was affected in living cells. To test this hypothesis, we have performed FRAP assay in ARPE-19 cells (Fig. 8). The cell line was transfected with the plasmids pEGFP-N1, PRPF31-GFP and A216P-GFP and FRAP was performed as described in the Methods section of this manuscript. Pre- and post-photobleached cells are displayed in Fig. 8a-l, showing that the amount of PRPF31 protein recovered in the nucleus $200 \mathrm{~s}$ after bleaching is affected by the mutation (Fig. 8l) but not in pEGFP-N1 (Fig. 8d) or PRPF31-GFP (Fig. 8h) transfected cells. A slower recovery curve for $A 216 P$-GFP were clearly observed when compared to the control PRPF31-GFP (Fig. $8 \mathrm{~m}$ ).

The changes produced in the recovery curve were due to changes in the size of $\mathrm{mf}$ and not due to changes in the $\tau_{1 / 2}$, which is defined as the time when the recovery of fluorescence intensity is half of the plateau $\left(\tau_{1 / 2}\right.$ PRPF31-GFP $=7.40 \pm 3.08 \mathrm{~s} ; \tau_{1 / 2}$ $A 216 P$-GFP $=7.00 \pm 2.66 \mathrm{~s} ; p=0.46$ ). However, the $\mathrm{mf}$ significantly decreased in A216P-GFP transfected cells $(\mathrm{mf}$ PRPF31-GFP $=0.55 \pm 0.10 ; \mathrm{mf}$ A216P-GFP $=$ $0.27 \pm 0.15 ; p<0.05)$ (Fig. $8 \mathrm{n}$ ). The $\mathrm{mf}$ was determined by comparing the fluorescence in the bleached region after full recovery $(F \infty)$ with the fluorescence before bleaching (Fi) and just after bleaching (F0), being defined as $\mathrm{mf}=(\mathrm{F} \infty-\mathrm{F} 0) /(\mathrm{Fi}-\mathrm{F} 0)$. The fluorescence intensity was normalized to the $\mathrm{Fi}$ and $\mathrm{FO}$ in the Fig. $8 \mathrm{~m}$. The A216P-GFP transfected cells showed less $\mathrm{F} \infty$ than the control (Fig. $8 \mathrm{~m}$ ). These results suggest that p.A216P mutation decreases the amount of PRPF31 protein that moves to the nucleus but without affecting the diffusion time.

\section{Discussion}

PRPF31 is a ubiquitously expressed gene encoding for a component of the spliceosome complex involved in premRNA processing. Mutations in this gene are associated with non-syndromic adRP, but the mechanism by which retinal degeneration occurs, is still unknown. Previously, two mutant mouse models (Prprf31 $1^{+/-}$and $\operatorname{Prpf} 31^{A 216 P /+}$ ) were generated to study the role of PRPF31 in the pathogenesis of adRP, but neither of these models presented evidences of RP-like photoreceptor degeneration, leading to conclude that the presence of one copy of WT-Prpf31 allele is sufficient to maintain the normal retina, and that the p.A216P mutation does not exert a dominant-negative effect (Bujakowska et al., 2009).

It has previously been described that the Prprf31 ${ }^{+/-} \mathrm{KO}$ mice (Farkas et al., 2014; Graziotto et al., 2011), as well as other splicing-factor mouse models that do not present photoreceptor-degenerative phenotype, have an RPEdegenerative phenotype. In this study, a deep phenotypic characterization of 8 to 16 month-old $\operatorname{Prpf} 31^{A 216 P /+}$ mice was performed to understand how the p.A216P mutation affects RPE function and we found evidence that supports a combination of haploinsufficiency and dominantnegative effect. Fundus analysis showed a severe RPE degeneration, with the presence of white-yellowish and autofluorescent spots in mutant mice. Concomitant functional impairment was detected in ERG c-wave. TEM images accordingly showed some typical features of degenerative RPE such as vacuolization, atrophy of basal infoldings, thickening of Bruch's membrane and accumulation of amorphous electrodense material within this membrane. Two previous studies (Farkas et al., 2014; Graziotto et al., 2011) reported that two-year-old $\operatorname{Prpf} 3^{T 494 M /+}$ and Prpf8 ${ }^{H 2309 P /+}$ and the one-year-old Prpf $31^{+/-}$mice had similar features to the ones that we have found in 8month-old Prpf $31^{A 216 P /+}$ mice. The earlier onset of RPE degeneration in $\operatorname{Prpf} 31^{A 216 P /+}$ might indicate a possible toxic effect of the p.A216P mutant protein.

In addition to the RPE atrophy, we have also observed accumulation of lipofuscin granules and drusen-like deposits of free cholesterol between the RPE and the Bruch's membrane or between both basal laminae. These deposits are similar to the basal linear deposits 


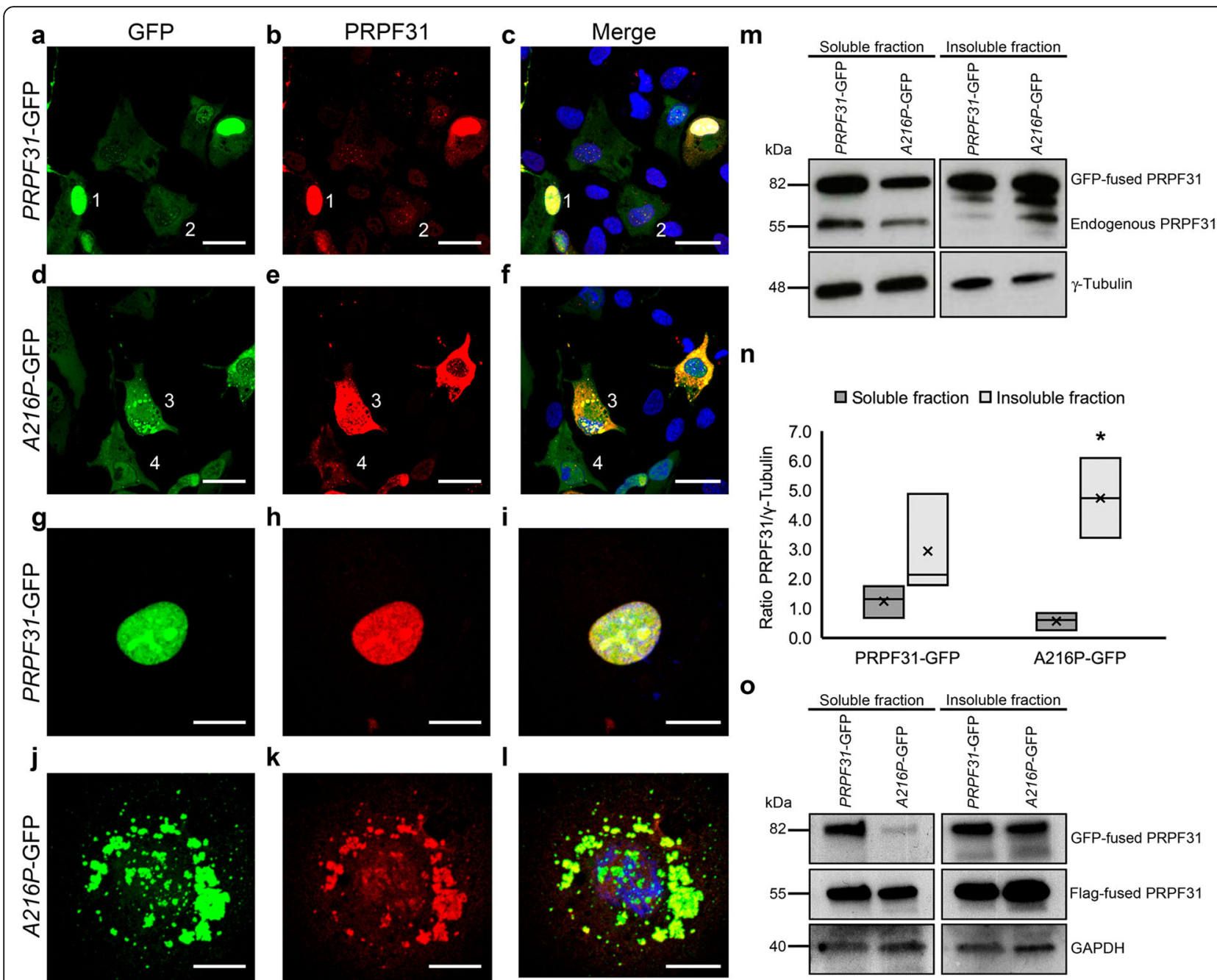

Fig. 6 Overexpression of A216P-GFP induces aggregation of PRPF31 protein. The ARPE-19 cell line was transfected with PRPF31-GFP (a-c, g-i) and A216P-GFP (d-fi, $\mathbf{j}-\mathbf{l})$. The GFP-tagged proteins (green) and immunostained cells with anti-PRPF31 antibodies (red) are shown. PRPF31-GFP was found mainly in the cell nucleus $(\mathbf{a}-\mathbf{c} ; \mathbf{1}, \mathbf{g}-\mathbf{i})$ and to a lesser extent, in the cytoplasm $(\mathbf{a}-\mathbf{c} ; \mathbf{2})$. A216P-GFP transfected cells present PRPF31 aggregation in the cytoplasm (d-f; $\mathbf{3}, \mathbf{j}-\mathbf{-})$, and a very minor signal in the nucleus $(\mathbf{d}-\mathbf{f} ; \mathbf{4})$. Images correspond to a maximum projection of a Zstack. Western blot analysis of soluble and insoluble fractions of the transfected cells show a decrease in the concentration of endogenous PRPF31 in the detergent-soluble fraction and an increase in the detergent-insoluble fraction of the cells transfected with A216P-GFP. Anti- - tubulin antibody was used as loading control $(\mathbf{m})$. Densitometry quantification of the blots (n) shows a significant increment of A216P-GFP protein in the detergent-insoluble fraction when compare with the among of A216P-GFP protein present in the soluble fraction (n). The boxplot $\mathbf{n}$ represents the ratio PRPF31/ $\gamma$-tubulin in the soluble and insoluble fractions of PRPF31-GFP and A216P-GFP transfected ARPE-19 cells ( $n=3$ in each group). Statistically significant differences were determined by Mann-Whitney $U$-test $\left.{ }^{*} p<0.05\right)$. Western blot analysis of soluble and insoluble fractions of PRPF31-GFP and A216P-GFP transfected ARPE-19 cells and co-transfected with WT PRPF31 tagged to Flag showed a decrease in the concentration of WT PRPF31-Flag in the detergent-soluble fraction and an increase in the detergent-insoluble fraction of the cells co-expressing the mutant A216P-GFP protein. Anti-GAPDH antibody was used as loading control (o). Scale bars (a-f) represent $25 \mu \mathrm{m}$ and $(\mathbf{g}-\mathrm{I}) 10 \mu \mathrm{m}$

and basal lamellar deposits described in AMD patients (Curcio \& Millican, 1999). RPE atrophy, lipofuscin accumulation and thickening of Bruch's membrane are characteristic histological hallmarks found in human AMD patients, and these features have been also described in animal models of RPE degeneration (Curcio \& Millican, 1999; Pennesi et al., 2012). Therefore, the Prpf31 $1^{A 216 P /+}$ mice display a primary RPE degeneration phenotype with drusen-like deposits. Although RP phenotype is predominantly associated with PRPF31 mutations in humans, some affected individuals developed juvenile macular degeneration apart from the typical RP phenotype, as described in two Chinese families carrying different mutations in the PRPF31 gene (Lu et al., 2013; Xi et al., 2005). These results suggest that mutations in the PRPF31 gene could produce not only a characteristic RP 


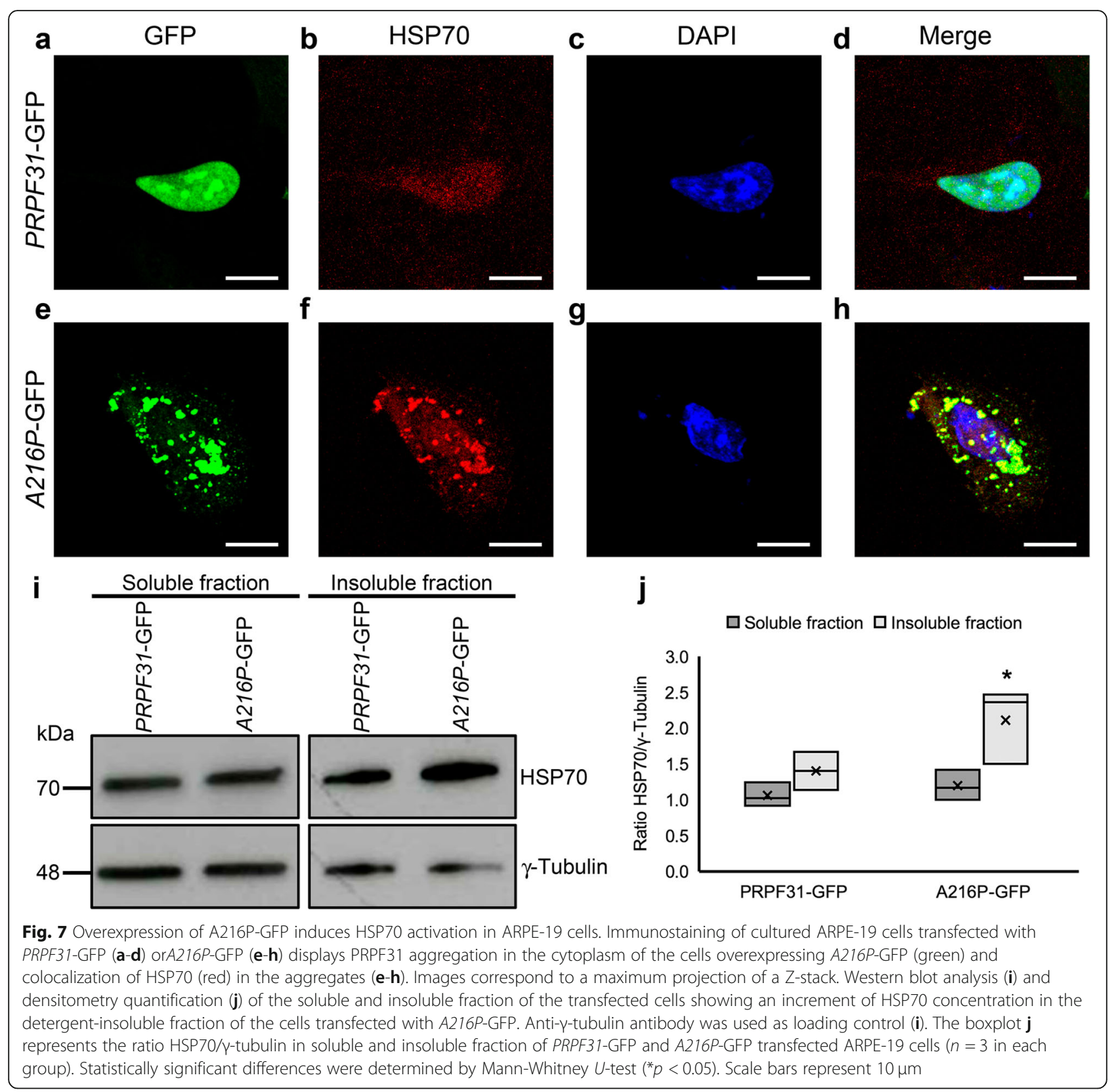

phenotype in humans but also a phenotype with an early macular degeneration. Similar results have been observed with mutations in another gene such as RDS, which encodes the photoreceptor glycoprotein peripherin. Mutations in RDS can produce both a clear RP phenotype and also macular dystrophy (Wells et al., 1993). Although no single nucleotide polymorphism in PRPF31 gene has been described in AMD, it has been suggested that alterations in normal mRNA splicing could contribute to the pathophysiology of age-related diseases such as AMD ( $\mathrm{Li}$ et al., 2017). In the Prpf31 mutant mice, we observed that the splicing of several genes is compromised. Among these genes, the $A B C A 4$ gene stands out. $A B C A 4$ is a gene expressed mainly in photoreceptors but also RPE having a major function in retinol metabolism (Lenis et al., 2018). Mutations in this gene have been found to cause Stargardt's disease, a hereditary juvenile macular degeneration, and AMD (Baum et al., 2003). Besides, it has been reported that $A B C A 4$ splicing can be also affected by aging and in AMD cases (Li et al., 2017; Baum et al., 2003; Meshorer \& Soreq, 2002). For these reasons, we suggest that primary RPE degeneration phenotype with drusen-like deposits present in these Prpf31 mutant mice could be due to splicing defects in genes that could be associated with macular degenerative diseases such as $A B C A 4$. 

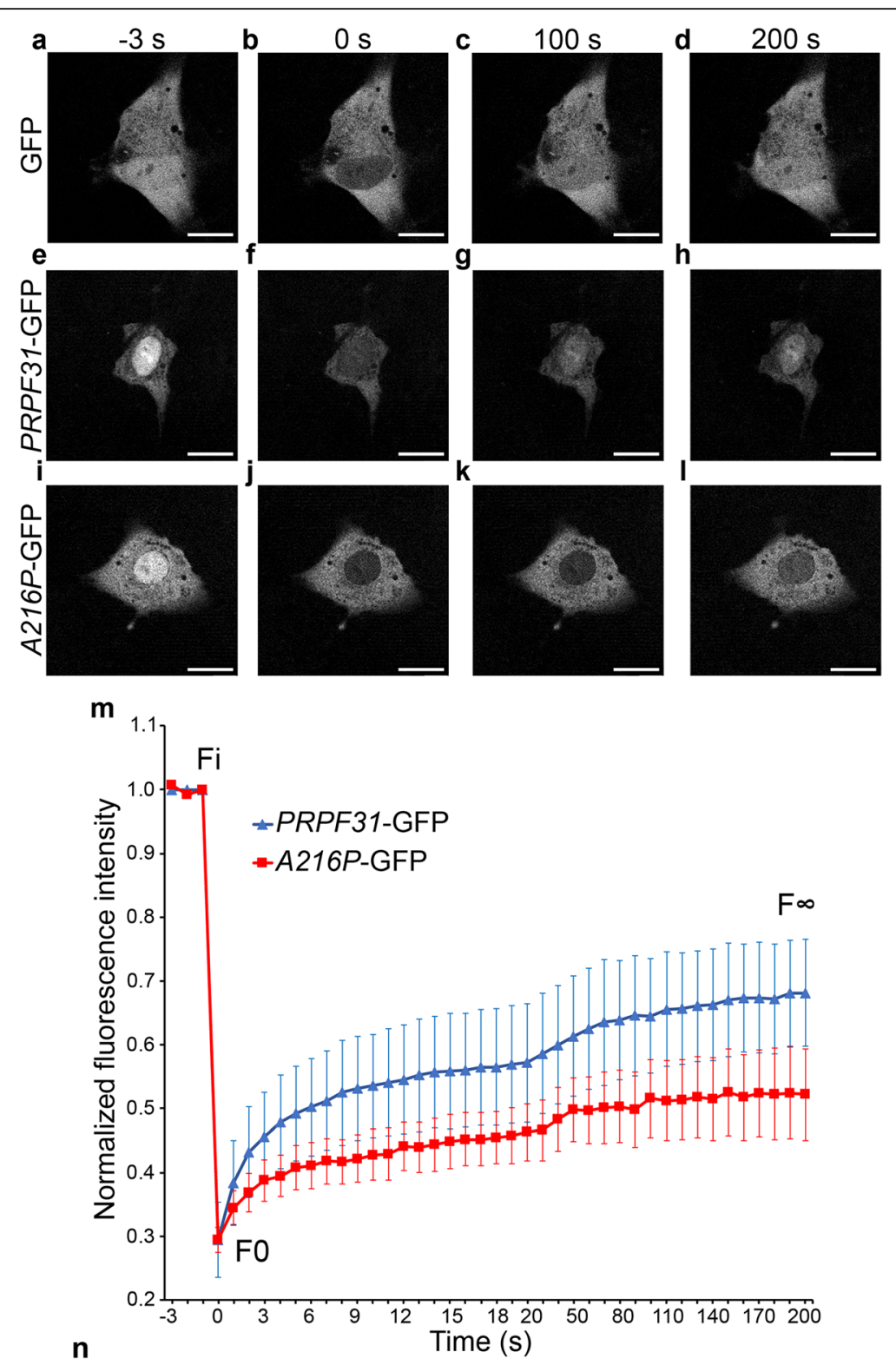

n

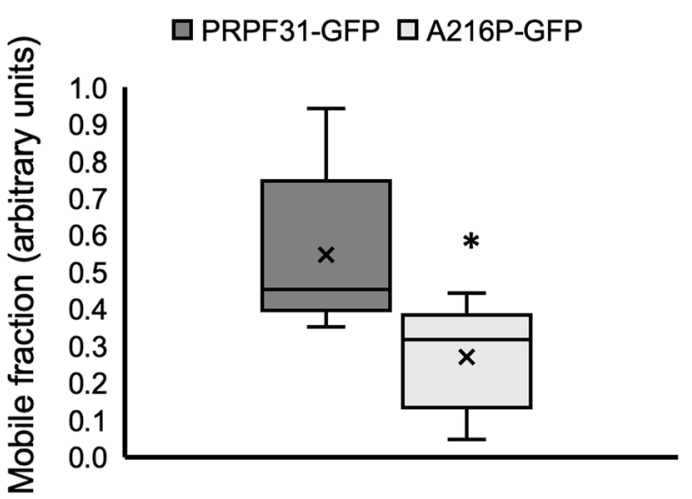

Fig. 8 (See legend on next page.) 
(See figure on previous page.)

Fig. 8 Mobility of PRPF31 protein to the nucleus is affected by the P.A216P mutation. ARPE-19 cells transfected with the pEGFP-N1 plasmid alone or carrying the open reading frame of human WT PRPF31 (PRPF31-GFP) or carrying the point mutation p.A216P (A216P-GFP) were imaged - $3 \mathrm{~s}$ pre-photobleaching $(\mathbf{a}, \mathbf{e}, \mathbf{i})$ and $0 \mathrm{~s}(\mathbf{b}, \mathbf{f}, \mathbf{j}), 100 \mathrm{~s}(\mathbf{c}, \mathbf{g}, \mathbf{k})$ and $200 \mathrm{~s}(\mathbf{d}, \mathbf{h}, \mathbf{l})$ post-photobleaching. The recovery curve (m) indicates that the cells transfected with mutant A216P-GFP have a smaller $\mathrm{mf}$ than those transfected with PRPF31-GFP $(\mathbf{n})$. The boxplot $\mathbf{n}$ represents the normalized fluorescence intensity and mobile fraction in PRPF31-GFP and A216P-GFP transfected ARPE-19 cells ( $n=5$ in each group). Statistically significant differences were determined by Mann-Whitney U-test $\left({ }^{*} p<0.05\right)$. Fi $=$ fluorescence before bleaching, $\mathrm{F} 0=$ fluorescence just after bleaching, F $\infty=$ fluorescence in the bleached region after full recovery. Scale bars represent $10 \mu \mathrm{m}$

It is known that the RPE maintains structural integrity and function of photoreceptor cells and defends the retina from free radicals and photo-oxidative damage (Simo et al., 2010; Strauss, 2005). A failure in any of the RPE roles might induce retinal degeneration and loss of visual function. Two classical examples of RP due to mutations in RPE-specific genes are MERTK and RPE65, which cause RP (Gal et al., 2000) and Leber's congenital amaurosis (Gu et al., 1997), respectively. Thus, p.A216P PRPF31 mutation might affect photoreceptors due to a primary defect in the RPE as MERTK or RPE65 mutations do.

As stated before, in humans, mutations in PRPF31 are associated with photoreceptor-cell degeneration and loss. The reason why $\operatorname{Prpf} 31^{A 216 P /+}$ mice have RPEdegeneration rather than photoreceptor-cell loss is not clear, but we have found that gene expression level for Prpf31 and the corresponding protein amount is much higher in the RPE than in the neuroretina, although the nuclei in the neuroretina greatly outnumber the nuclei in the RPE monolayer. This was also observed in mouse, pig, cow and human samples. Yuan and co-workers have studied the expression of Prpf31 in different mouse tissues including the retina (Yuan et al., 2005). Although no differences were observed either in the expression level or pattern of Prpf31 protein among different tissues, they did not separate the neuroretina from the RPE as we have done in this study and in fact, their in situ hybridization results clearly show a much higher expression of Prpf31 in the RPE layer. The differential expression of Prpf31 could give us clues to understand why the RPE specifically degenerates in these mice. The elevated requirement of Prpf31 protein level in the RPE could be related to high or specific splicing demands in an epithelium that fulfils many different roles: light absorption, transport of ions, water, and metabolic end-products from the subretinal space to blood, maintenance of photoreceptors and re-isomerization of all-trans-retinal into 11-cis-retinal. Thus, defects in splicing might have a great impact on the normal function and survival of RPE cells.

To explore the role of Prpf31 p.A216P mutation in the $\mathrm{RPE}$, the distribution pattern of the protein was analysed by immunofluorescence staining of whole-mount RPE samples and Western blot of cytosolic and nuclear fractions from WT and Prpf31 $1^{A 216 P /+}$ mutant mice. As expected, we found Prpf31 preferentially localized in the nucleus of RPE cells in WT mice, although it was also possible to observe a cytoplasmic fraction. On the other hand, in the mutant mice almost no Prpf31 was found within the nucleus, and most of the Prpf31 staining was present in the cytoplasm forming rounded clumps of Prpf31 protein resembling aggregates. The distribution of RPE cells having Prpf31 aggregates was not uniform, and we do not have an explanation for this finding. Nevertheless, our results are in accordance with the ones published by Deery and co-workers (Deery et al., 2002), in which they found that overexpression of A194E and A216P mutations in COS-7 cells induced mislocalization of the mutant PRPF31 proteins, distributed throughout the cytoplasm and with less intense staining in the nucleus when compared with WT-PRPF31 transfected cells. Also, Huranová and colleagues described similar results overexpressing A216P in HeLa cells. They found mislocalization of the mutant protein causing depletion of PRPF31 from Cajal bodies, where splicing takes place, and described a possible negative effect due to an abnormal interaction of A216P with its partner, PRPF6 (Huranova et al., 2009). It is important to note, that the Prpf31 protein detected in these mice corresponds to the endogenous WT and mutant forms of the protein. Thus, the aggregation that is found in the RPE of these mutant mice is not an artefact resulting from Prpf31 overexpression. In $\operatorname{Prpf} 31^{A 216 P /+}$ mice, aggregation of the mutant protein completely depletes Prpf31 protein in the nucleus and, in this way, a combination of a dominantnegative effect and haploinsufficiency might contribute to RPE degeneration.

Although the exact mechanism underlying RPE degeneration in the mutant mice is not well understood yet, our transcriptomic analysis show that $1.6 \%$ of evaluated genes are differentially expressed in the mutant RPE when compared to WT controls. As expected, alternative splicing was also affected in the RPE of these mutant mice. Around $11 \%$ of evaluated genes have, at least, one differentially expressed probe selection region or junction, indicative of alternative splicing. From the pool of affected genes, we selected $H s p a 4 l$ for a detailed study, a member of the HSP70 family of chaperons that was upregulated in the mutant RPE. The HSP70 family is a ubiquitous and conserved family of molecular chaperons, 
part of the cellular machinery for protein folding that prevents aggregation and protects cells from stress (Mashaghi et al., 2016; Furukawa \& Koriyama, 2016). Several reports have shown that HSP70 and also small heat shock proteins play an important role in retinal dystrophies, including RP (Saliba et al., 2002), glaucoma (Nagashima et al., 2011; Park et al., 2001; Schallenberg et al., 2012) and AMD (Alge et al., 2002; Lee et al., 2011; Nakata et al., 2005). Our qPCR analysis showed increased expression of Hspa4l in the mutant RPE when compared with its WT control. We further confirmed this finding by Western blot analysis depicting a higher amount of protein in the mutant RPE. Moreover, immunofluorescence staining showed colocalization of Hspa4l signal with aggregates of Prpf31 protein in the RPE cytoplasm of mutant mice. This suggests that Hspa4l can be acting as a chaperone for the mutant protein in response to its aggregation. However, the aggregation of Prpf 31 in the RPE of mutant mice could also activate other chaperones belonging to the family of small heat shock proteins such as Hsp27.

In addition, several other genes and signalling pathways were found to have an altered splicing in the RPE of mutant mice, including those associated with inflammation, oxidative stress, retinol metabolism and cellular apoptosis Most of these pathways are commonly affected in RPE degenerative diseases such as AMD and mutations of some genes including in this pathways such as $A B C A 4$ cause macular degeneration (Lenis et al., 2018; Makarev et al., 2014). In line with these results, our histological findings in the mutant RPE correlate with some macular degenerative features. Apart from that, another group of genes affected by the A216P mutation in RPE are involved in ciliogenesis. This result is in agreement with the most recent results of Buskin and co-workers in which they show that iPS-derived RPE cells from RP11 patients present shorter $\mathrm{Mv}$ and primary cilia, loss of polarity, reduced barrier function and defective phagocytic capacity, when compared to iPS-derived RPE cells from healthy donors, suggesting that these distorted cellular characteristics result from alternative splicing in RP11 (Buskin et al., 2018). We observed that one group of proteins affected by differential splicing in Prpf31 $1^{A 216 P /+}$ mice are some splicing factors, including Prpf31. Four main causes are known to induce protein aggregation: mutations, errors in protein synthesis including splicing defects, environmental factors such as oxidative stress and aging. Therefore, if the Prpf31-protein level in the RPE nucleus is beneath its threshold for normal function, the mRNA splicing of Prpf31 gene could be affected producing an aberrant protein prone to aggregation, reinforcing the dominant-negative effect and haploinsufficiency.
To explore the role of Hsp70 family in the RPE affected by a p.A216P mutation, we overexpressed both WT PRPF31 and mutant A216P tagged to GFP in a humanderived RPE cell line (ARPE-19). Immunofluorescence staining results are in line with the in vivo results, with WT PRPF31-GFP protein being located mainly in the nucleus, and mutant $A 216 P$-GFP protein being mostly aggregated in the cytoplasm. By Western blot analysis we found that cells overexpressing the mutant variant have a significant decrease in the soluble fraction of PRPF31 and an increase in the insoluble PRPF31 protein. Moreover, the overexpression of the mutant protein leads to a depletion of the soluble endogenous PRPF31 protein and WT PRPF31 tagged to Flag as well, suggesting a dominantnegative effect. These results are in accordance with one of the mechanisms proposed by Yin and coworkers in which they report that mutations in PRPF31 can induce retinal degeneration (Yin et al., 2011). They proposed three mechanisms: i) haploinsufficiency due to loss of function of the mutant protein or degradation of mutant mRNA by nonsense-mediated mRNA decay, thus compromising the splicing machinery; ii) mutant proteins with dominant-negative activity that may interfere with splicing and potentially with other cellular activities, leading to degeneration of the affected tissue; and iii) mutations might promote proteins forming insoluble and cytotoxic aggregates that can affect the tissue by loss-of-function and dominant-negative effects (Yin et al., 2011). We also observed that Hsp70 was upregulated in the cells overexpressing A216P-GFP and colocalized with PRPF31 mutant protein. These results corroborate our in vivo findings of Hspa4l upregulation in the RPE of Prpf $31^{A 216 P /+}$ mice and its colocalization with PRPF31 protein aggregates.

Considering that PRPF31 plays its role in the nucleus and p.A216P induces PRPF31 aggregation in the cytoplasm of RPE cells, we decided to analyze how PRPF31 translocation to the nucleus was affected using FRAP assay. PRPF31 contains a classical nuclear localization sequence (NLS) between residues 351 and 364. In a previous study published by Wilkie, et al. (Wilkie et al., 2006) demonstrated that p.A216P mutations did no affect the interaction with importin $\beta 1$. However, FRAP studies in the green monkey kidney fibroblast-like COS-7 cells of WT PRPF31-GFP and mutant A216P-GFP transfected cells indicated the presence of two-component recovery processes, a fast component for free diffusion within an unbounded compartment and a slow component for passive diffusion through the nuclear pores. The kinetics of both components were not affected by the p.A216P mutation (Wilkie et al., 2006). Therefore, seems that the mutation p.A216P did not affect the NLS and its interaction with importin $\beta 1$ but the total amount of nuclear PRPF31 is affected probably because there is lest amount of cytoplasmic PRPF31 that can cross the nuclear pores through 
passive diffusion. We conclude in our FRAP experiments that p.A216P mutation decreases the amount of PRPF31 protein that moves to the nucleus but without affecting the diffusion time. We found that translocation of $\mathrm{mf}$ of PRPF31 to the nucleus significantly decreases in ARPE-19 cells transfected with A216P-GFP. Our results are partially in line with the studies by Wilkie and co-workers who showed a similar FRAP pattern in COS-7 cells, although they could not find a statistically significant delay in mutant protein translocation to the nucleus when compared with the WT protein (Wilkie et al., 2006). This can be an artifact caused by the cell line, since it has been shown that impaired splicing due to PRPF31 mutations is limited to retinal cells (Buskin et al., 2018). These authors have reported that alternative spliced transcripts due to RP11 are only present in retina and RPE of the Prpf $31^{+/-}$mutant mice but not in other tissues such as brain or muscle suggesting that a specific and highly regulated splicing program takes place in the retinal cells. One of the most interesting findings of these authors (Buskin et al., 2018) with respect to our study is the fact that some alternatively spliced forms of PRPF31 were found in RPE but not in the neuroretina of the mutant $\mathrm{KO}$ mouse, supporting our finding that lack of PRPF31 in the nuclei of RPE cells could affect its own splicing.

\section{Conclusion}

Our findings of a substantial higher amount of PRPF31 in the RPE compared to the neuroretina, exhibiting a degenerative phenotype of the RPE with drusen-like deposits, correlates with the growing amount of data supporting a specific splicing program in retinal cell types that is severely distorted by PRPF31 mutations. Our results suggest that p.A216P mutation promotes a damaging alternative splicing of PRPF31, generating a dominant-negative effect in which aberrant splicing of other genes and PRPF31 mutated protein aggregation are involved. Depletion of functional PRPF31 protein from the nucleus will also contribute to deficient splicing, thus mixing haploinsufficiency with a dominantnegative effect. In response to protein aggregation of PRPF31, HSPA4L chaperon is activated and recruited to the aggregates, to fold the aggregated protein and facilitate its translocation to the nucleus. A pharmacological or genetic modulation of HSP70 family of proteins could be a promising therapeutic target for RP caused by PRPF31 mutations.

\section{Supplementary information}

Supplementary information accompanies this paper at https://doi.org/10. 1186/s10020-019-0124-z.

Additional file 1: Figure S1. TEM images of RPE of 8-month-old WT (a) and $\operatorname{Prpf} 33^{A 216 P /+}$ mice (b). Photoreceptor outer segments (OS) were observed in contact with the RPE microvilli (Mv) in WT mice (a). In $\operatorname{Prpf3} 1^{\mathrm{A} 216 \mathrm{P} / \mathrm{+}}$ mice OS and Mv are also observed; however, they looked shorter and disorganized (b). RPE nuclei (Nu), melanin granules (Me), basal infoldings (BI), Bruch's membrane (BM) and choroid (Co) are also displayed. Scale bars represent $5 \mu \mathrm{m}$. Figure S2. ERG recordings of WT and Prpf3 $1^{\mathrm{A216P/+}}$ mice. Rod response and quantification of b-wave amplitude in dark adapted mice (a-a"). Cone-rod response and quantification of a-wave in dark adapted mice ( $\left.\mathbf{b}-\mathbf{b}^{\prime \prime}\right)$. Cone response and quantification of b-wave in light adapted mice $\left(\mathbf{c}-\mathbf{c}^{\prime \prime}\right)$. RPE response and quantification of c-wave in dark adapted mice $\left(\mathbf{d}-\mathbf{d}^{\prime \prime}\right)$. The boxplots $\mathbf{a}^{\prime \prime}-\mathbf{d}$ " represent the $\mathrm{a}, \mathrm{b}$ and $\mathrm{c}$-wave amplitude in dark or light adapted WT and Prpf31 $1^{\mathrm{A2} 16 \mathrm{P} /+}$ mice ( $n=6$ in each group). Statistically significant differences were determined by Mann-Whitney $U$-test $\left({ }^{*} p<0.05\right)$. Vertical scale bars represent $300 \mu \mathrm{V}$. Horizontal scale bars represent $100 \mathrm{~ms}\left(\mathbf{a}-\mathbf{c}^{\prime}\right)$ and $1 \mathrm{~s}\left(\mathbf{d}-\mathbf{d}^{\prime}\right)$. Figure S3. Western blot analyses of PRPF31 expression in the neuroretina and RPE of C57BL/6 mice, pig, cow and human samples. The amount of PRPF31 protein is higher in the RPE than in the neuroretina in all the cases. Anti-y-tubulin antibody was used as loading control. Figure S4. Whole-mount of the RPE obtained from WT (a-i) and Prpf3 $1^{\text {A216P/+ }}$ mice $(\mathbf{j}-\mathbf{I})$. Negative controls of RPE autofluorescence $(\mathbf{a}-\mathbf{c})$ and secondary antibodies nonspecific bindings are shown (d-f). The RPE of WT Prpf31 $1^{\text {A216P/+ }}$ mice were immunostained with anti-Prpf31 $(\mathbf{g}, \mathbf{j})$ and anti-Hsp27 antibodies (h, k). TRITC-phalloidin was used to stain F-actin microfilaments (a-l; blue). Merged images are shown (c, f, i, l). Prpf31 protein aggregates were observed in the cytoplasm colocalizing with Hsp27 signal in mutant Prpf3 $1^{\text {A216P/+ }}$ mice $(\mathbf{j}-\mathbf{l})$. Z-stack of a ARPE-19 cell transfected with PRPF31GFP ( $\mathbf{m}-\mathbf{o})$. In the Z-stacks is possible to observe that PRPF31-GFP signal is in the nucleus and not in the top of the nucleus. Scale bars represent $25 \mu \mathrm{m}$. Table S1. Gene ontology of differentially expressed genes in Prpf31A216P/+ mice. The top 20 terms of 84 are listed. Table S2. Candidate genes differentially expressed in the RPE of Prpf31A216P/+ vs WT mice. Default filter criteria, fold change $<-2$ or $>2$ and $p$-value $<0.05$. Table S3. Functional categories of genes having alternative splicing in Prpf31A216P/+ mice. The top 40 terms of 174 are listed. Table S4. Number of candidate genes showing alternative splicing in the RPE of Prpf31A216P/+ vs WT mice. Default filter criteria, splicing index $<-2$ or $>2$ and ANOVA $p$ value $<0.05$.

Additional file 2. Results of transcriptome array (MTA) 1.0 to evaluate differential gene expression in RPE samples of six Prpf31 $1^{A 216 P /+}$ and three WT-littermates.

Additional file 3. Results of alternative splicing analysis (MTA) 1.0 in RPE samples of six Prpf3 $1^{A 216 P /+}$ and three WT-littermates.

\section{Abbreviations}

adRP: Autosomal dominant retinitis pigmentosa; AMD: Age-related macular degeneration; cd: Candela; CT: Average cycle threshold; DAVID: Database for Annotation, Visualization and Integrated Discovery; ERG: Electroretionogram; F0: Fluorescence just after bleaching; Fo: Fluorescence in the bleached region after full recovery; Fi: Fluorescence before bleaching;

FRAP: Fluorescence recovery after bleaching; HSP70: Heat shock protein 70; Hspa4l: Heat shock $70 \mathrm{kDa}$ protein $4 \mathrm{~L} ;$ Hspa4l: Heat shock protein family $A$ (Hsp70) member 4 like gene; Kl: Knock-in; KO: Knockout; mf: Mobile fraction; MTA 1.0: Mouse Transcriptome Array; Mv: Microvilli; NLS: Nuclear localization sequence; OS: Outer segments; PBS-T: 0.2\% Triton X-100/PBS;

PFA: Paraformaldehyde; qPCR: Quantitative RT-PCR; RP: Retinitis pigmentosa; RPE: Retinal pigment epithelium; TEM: Transmission electron microscopy; $\tau_{1 / 2}$ : Half-life

\section{Acknowledgements}

The authors thank Paloma Domínguez Giménez for excellent technical assistance.

\section{Authors' contributions}

SMC, AGD and AMS performed the in vitro studies. LVS and SMC analyzed the data and wrote the manuscript. LVS and AA performed the in vivo studies and histological experiments. BC and ERB performed the proteomic studies. BC reviewed de manuscript. AA analyzed the in vivo experiments. SM performed the cloning and site-mutagenesis of PRPF31. VB performed the RPE whole mounts. ADL and DRM performed the GPCR. SSB and FDC designed, supervised and analyzed the experiments, as well as reviewed the 
manuscript and obtained the funding. All authors read and approved the final manuscript.

\section{Funding}

This project has been financed through a) The ISCIII (Miguel Servet-I, 2015), co-financed by the European Regional Development Fund (ERDF), No CP15/ 00071. b) The European Union's Horizon 2020 research and innovation program, under grant agreement No 634479. c) Regional Ministry of Economy, Innovation and Science of the Junta de Andalucía, No P09-CTS-04967.

\section{Availability of data and materials}

All data generated or analyzed during this study are included in this published article, in the supplementary files.

\section{Ethics approval and consent to participate}

The present work used human and animal samples. All experiments described herein were performed in compliance with the Spanish and European Laboratory Animal Science Association-FELASA Guide for the Care and Use of Laboratory Animals, the European Union Council Directive 2010/ 63/EU for the use of animals and the Association for Research in Vision and Ophthalmology-ARVO for the use of animals in ophthalmic and vision research. Animal manipulation and experimental methods have been approved by the Ethics Committee for Animal Experimentation of CABIMER, Seville, Spain. All efforts were made to minimize the number of animals used and their suffering. Pig and cow eye samples were obtained from a local slaughterhouse. Human eye sample for Western blotting was obtained from a deceased healthy donor, in a procedure approved by the Ethics Committee of University Hospital Virgen Macarena, Seville, Spain.

\section{Consent for publication}

No individual data to disclose.

\section{Competing interests}

The authors declare that they have no competing interests.

\section{Author details}

${ }^{1}$ Regeneration and Cell Therapy Department, Andalusian Molecular Biology and Regenerative Medicine Centre-CABIMER (Junta de Andalucía), CSIC, Universidad de Sevilla, Universidad Pablo de Olavide, Avda. Americo Vespucio 24, 41092 Seville, Spain. ${ }^{2}$ Present Address: Center for Biomedical Research (CBMR), University of Algarve, 8800-139 Faro, Portugal. ${ }^{3}$ Present Address: Clinique de I'Oeil, Avenue Bois de la Chapelle 15, 1213 Onex, Switzerland. ${ }^{4}$ Present Address: Center for Molecular and Cellular Bioengineering (CMCB) DFG-Research Center for Regenerative Therapies Dresden (CRTD) Cluster of Excellence, Technische Universität Dresden, Fetscherstraße, 10501307 Dresden, Germany. ${ }^{5}$ Present Address: Universitätsklinikum Tübingen, Forschungsinstitut für Augenheilkunde, Elfriede-Aulhorn-Str. 7, 72076 Tübingen, Germany. ${ }^{6}$ Institute of Ophthalmology, University College London, 11-43 Bath Street, London EC1V 9EL, UK.

Received: 30 May 2019 Accepted: 5 December 2019

Published online: 31 December 2019

\section{References}

Alge CS, Priglinger SG, Neubauer AS, Kampik A, Zillig M, Bloemendal H, et al. Retinal pigment epithelium is protected against apoptosis by alphaBcrystallin. Invest Ophthalmol Vis Sci. 2002;43(11):3575-82.

Al-Maghtheh M, Vithana EN, Inglehearn CF, Moore T, Bird AC, Bhattacharya SS. Segregation of a PRKCG mutation in two RP11 families. Am J Hum Genet. 1998;62(5):1248-52.

Baum L, Chan WM, Li WY, Lam DS, Wang PB, Pang CP. ABCA4 sequence variants in Chinese patients with age-related macular degeneration or Stargardt's disease. Ophthalmologica. 2003;217(2):111-4.

Bujakowska K, Maubaret C, Chakarova CF, Tanimoto N, Beck SC, Fahl E, et al. Study of gene-targeted mouse models of splicing factor gene Prpf31 implicated in human autosomal dominant retinitis pigmentosa (RP). Invest Ophthalmol Vis Sci. 2009;50(12):5927-33.

Buskin A, Zhu L, Chichagova V, Basu B, Mozaffari-Jovin S, Dolan D, et al. Disrupted alternative splicing for genes implicated in splicing and ciliogenesis causes PRPF31 retinitis pigmentosa. Nat Commun. 2018;9(1):4234.
Curcio CA, Millican CL. Basal linear deposit and large drusen are specific for early age-related maculopathy. Arch Ophthalmol. 1999;117(3):329-39.

Deery EC, Vithana EN, Newbold RJ, Gallon VA, Bhattacharya SS, Warren MJ, et al. Disease mechanism for retinitis pigmentosa (RP11) caused by mutations in the splicing factor gene PRPF31. Hum Mol Genet. 2002;11(25):3209-19.

Diaz-Corrales FJ, Asanuma M, Miyazaki I, Miyoshi K, Ogawa N. Rotenone induces aggregation of gamma-tubulin protein and subsequent disorganization of the centrosome: relevance to formation of inclusion bodies and neurodegeneration. Neuroscience. 2005;133(1):117-35.

Ding X, Patel M, Chan CC. Molecular pathology of age-related macular degeneration. Prog Retin Eye Res. 2009;28(1):1-18.

Farkas MH, Lew DS, Sousa ME, Bujakowska K, Chatagnon J, Bhattacharya SS, et al. Mutations in pre-mRNA processing factors 3, 8, and 31 cause dysfunction of the retinal pigment epithelium. Am J Pathol. 2014;184(10):2641-52.

Furukawa A, Koriyama Y. A role of heat shock protein 70 in photoreceptor cell death: potential as a novel therapeutic target in retinal degeneration. CNS Neurosci Ther. 2016:22(1):7-14.

Gal A, Li Y, Thompson DA, Weir J, Orth U, Jacobson SG, et al. Mutations in MERTK, the human orthologue of the RCS rat retinal dystrophy gene, cause retinitis pigmentosa. Nat Genet. 2000;26(3):270-1.

Graziotto JJ, Farkas MH, Bujakowska K, Deramaudt BM, Zhang Q, Nandrot EF, et al. Three gene-targeted mouse models of RNA splicing factor RP show late-onset RPE and retinal degeneration. Invest Ophthalmol Vis Sci. 2011;52(1):190-8.

Gu SM, Thompson DA, Srikumari CR, Lorenz B, Finckh U, Nicoletti A, et al. Mutations in RPE65 cause autosomal recessive childhood-onset severe retinal dystrophy. Nat Genet. 1997;17(2):194-7.

Huranova M, Hnilicova J, Fleischer B, Cvackova Z, Stanek D. A mutation linked to retinitis pigmentosa in HPRP31 causes protein instability and impairs its interactions with spliceosomal snRNPs. Hum Mol Genet. 2009;18(11):2014-23.

Irizarry RA, Hobbs B, Collin F, Beazer-Barclay YD, Antonellis KJ, Scherf U, et al. Exploration, normalization, and summaries of high density oligonucleotide array probe level data. Biostatistics. 2003:4(2):249-64.

Lee $H$, Chung $H$, Lee $\mathrm{SH}$, Jahng WJ. Light-induced phosphorylation of crystallins in the retinal pigment epithelium. Int J Biol Macromol. 2011; 48(1):194-201.

Lenis TL, Hu J, Ng SY, Jiang Z, Sarfare S, Lloyd MB, et al. Expression of ABCA4 in the retinal pigment epithelium and its implications for Stargardt macular degeneration. Proc Natl Acad Sci U S A. 2018;115(47):E11120-7.

Li H, Wang Z, Ma T, Wei G, Ni T. Alternative splicing in aging and age-related diseases. Trans Med Aging. 2017;1:32-40.

Liu MM, Zack DJ. Alternative splicing and retinal degeneration. Clin Genet. 2013; 84(2):142-9.

Lu F, Huang L, Lei C, Sha G, Zheng H, Liu X, et al. A novel PRPF31 mutation in a large Chinese family with autosomal dominant retinitis pigmentosa and macular degeneration. PLoS One. 2013;8(11):e78274.

Makarev E, Cantor C, Zhavoronkov A, Buzdin A, Aliper A, Csoka AB. Pathway activation profiling reveals new insights into age-related macular degeneration and provides avenues for therapeutic interventions. Aging. 2014;6(12):1064-75.

Makarova OV, Makarov EM, Liu S, Vornlocher HP, Luhrmann R. Protein 61K, encoded by a gene (PRPF31) linked to autosomal dominant retinitis pigmentosa, is required for U4/U6*U5 tri-snRNP formation and pre-mRNA splicing. EMBO J. 2002;21(5):1148-57.

Mashaghi A, Bezrukavnikov S, Minde DP, Wentink AS, Kityk R, Zachmann-Brand B, et al. Alternative modes of client binding enable functional plasticity of Hsp70. Nature. 2016;539(7629):448-51.

Mattapallil MJ, Wawrousek EF, Chan CC, Zhao H, Roychoudhury J, Ferguson TA, et al. The Rd8 mutation of the Crb1 gene is present in vendor lines of C57BL/6N mice and embryonic stem cells, and confounds ocular induced mutant phenotypes. Invest Ophthalmol Vis Sci. 2012;53(6):2921-7.

Mayer MP, Bukau B. Hsp70 chaperones: cellular functions and molecular mechanism. Cell Mol Life Sci : CMLS. 2005;62(6):670-84.

Meshorer E, Soreq H. Pre-mRNA splicing modulations in senescence. Aging Cell. 2002;1(1):10-6.

Nagashima M, Fujikawa C, Mawatari K, Mori Y, Kato S. HSP70, the earliest-induced gene in the zebrafish retina during optic nerve regeneration: its role in cell survival. Neurochem Int. 2011;58(8):888-95.

Nakata K, Crabb JW, Hollyfield JG. Crystallin distribution in Bruch's membranechoroid complex from AMD and age-matched donor eyes. Exp Eye Res. 2005;80(6):821-6 
Park KH, Cozier F, Ong OC, Caprioli J. Induction of heat shock protein 72 protects retinal ganglion cells in a rat glaucoma model. Invest Ophthalmol Vis Sci. 2001;42(7):1522-30.

Pennesi ME, Neuringer M, Courtney RJ. Animal models of age related macular degeneration. Mol Asp Med. 2012;33(4):487-509.

Phair RD, Misteli T. Kinetic modelling approaches to in vivo imaging. Nat Rev Mol Cell Biol. 2001;2(12):898-907.

Reits EA, Neefjes JJ. From fixed to FRAP: measuring protein mobility and activity in living cells. Nat Cell Biol. 2001;3(6):E145-7.

Rose AM, Shah AZ, Venturini G, Krishna A, Chakravarti A, Rivolta C, et al. Transcriptional regulation of PRPF31 gene expression by MSR1 repeat elements causes incomplete penetrance in retinitis pigmentosa. Sci Rep. 2016;6:19450.

Ruzickova S, Stanek D. Mutations in spliceosomal proteins and retina degeneration. RNA Biol. 2017:14(5):544-52.

Saliba RS, Munro PM, Luthert PJ, Cheetham ME. The cellular fate of mutant rhodopsin: quality control, degradation and aggresome formation. J Cell Sci. 2002;115(Pt 14):2907-18.

Schaffert N, Hossbach M, Heintzmann R, Achsel T, Luhrmann R. RNAi knockdown of hPrp31 leads to an accumulation of U4/U6 di-snRNPs in Cajal bodies. EMBO J. 2004;23(15):3000-9.

Schallenberg M, Prokosch V, Thanos S. Regulation of retinal proteome by topical antiglaucomatous eye drops in an inherited glaucoma rat model. PLoS One. 2012;7(7):e33593.

Huang da W, Sherman BT, Lempicki RA. Systematic and integrative analysis of large gene lists using DAVID bioinformatics resources. Nat Protoc 2009;4(1): 44-57.

Simo R, Villarroel M, Corraliza L, Hernandez C, Garcia-Ramirez M. The retinal pigment epithelium: something more than a constituent of the blood-retinal barrier--implications for the pathogenesis of diabetic retinopathy. J Biomed Biotechnol. 2010;2010:190724

Strauss O. The retinal pigment epithelium in visual function. Physiol Rev. 2005; 85(3):845-81.

Verbakel SK, van Huet RAC, Boon CJF, den Hollander Al, Collin RWJ, Klaver CCW, et al. Non-syndromic retinitis pigmentosa. Prog Retin Eye Res. 2018:S13509462(17)

Vithana EN, Abu-Safieh L, Allen MJ, Carey A, Papaioannou M, Chakarova C, et al. A human homolog of yeast pre-mRNA splicing gene, PRP31, underlies autosomal dominant retinitis pigmentosa on chromosome 19q13.4 (RP11). Mol Cell. 2001;8(2):375-81

Vithana EN, Abu-Safieh L, Pelosini L, Winchester E, Hornan D, Bird AC, et al. Expression of PRPF31 mRNA in patients with autosomal dominant retinitis pigmentosa: a molecular clue for incomplete penetrance? Invest Ophthalmol Vis Sci. 2003:44(10):4204-9.

Wells J, Wroblewski J, Keen J, Inglehearn C, Jubb C, Eckstein A, et al. Mutations in the human retinal degeneration slow (RDS) gene can cause either retinitis pigmentosa or macular dystrophy. Nat Genet. 1993;3(3):213-8.

Wilkie SE, Morris KJ, Bhattacharya SS, Warren MJ, Hunt DM. A study of the nuclear trafficking of the splicing factor protein PRPF31 linked to autosomal dominant retinitis pigmentosa (ADRP). Biochim Biophys Acta. 2006;1762(3): 304-11.

Xi XH, Zheng D, Xia K, Pan Q, Lei LY, Liu Z, et al. Splicing site mutation of D19S418 in PRPF-31 gene and its phenotypic characters with autosomal dominant retinitis pigmentosa. Zhonghua Yan Ke Za Zhi. 2005;41(11):1020-6.

Yin J, Brocher J, Fischer U, Winkler C. Mutant Prpf31 causes pre-mRNA splicing defects and rod photoreceptor cell degeneration in a zebrafish model for retinitis pigmentosa. Mol Neurodegener. 2011;6:56.

Yuan L, Kawada M, Havlioglu N, Tang H, Wu JY. Mutations in PRPF31 inhibit premRNA splicing of rhodopsin gene and cause apoptosis of retinal cells. J Neurosci. 2005;25(3):748-57.

\section{Publisher's Note}

Springer Nature remains neutral with regard to jurisdictional claims in published maps and institutional affiliations.

Ready to submit your research? Choose BMC and benefit from:

- fast, convenient online submission

- thorough peer review by experienced researchers in your field

- rapid publication on acceptance

- support for research data, including large and complex data types

- gold Open Access which fosters wider collaboration and increased citations

- maximum visibility for your research: over $100 \mathrm{M}$ website views per year

At BMC, research is always in progress.

Learn more biomedcentral.com/submissions 